\title{
Chemical interdiffusion in binary systems; interface barriers and phase competition
}

\author{
Marek Danielewski, ${ }^{1}$ Bartek Wierzba, ${ }^{1, a)}$ Andriy Gusak, ${ }^{2}$ M. Pawełkiewicz,,${ }^{1,3}$ \\ and J. Janczak-Rusch ${ }^{3}$ \\ ${ }^{1}$ Interdisciplinary Centre for Materials Modeling, FMSci\&C, AGH University of Science and Technology, \\ Al. Mickiewicza 30, 30-059 Kraków, Poland \\ ${ }^{2}$ Department of Theoretical Physics, Cherkasy National University, Shevchenko Street 81, \\ Cherkasy 18000 Ukraine \\ ${ }^{3}$ Empa, Swiss Federal Laboratories for Materials Science and Technology, \\ Überlandstrasse 129, 8600 Dübendorf, Switzerland
}

(Received 9 February 2011; accepted 14 November 2011; published online 20 December 2011)

The problem of simultaneous growth and competition of intermediate phases during reactive diffusion is formulated and solved. In this paper, we compare existing models of steady state reaction diffusion and introduce the new one basing on the bi-velocity method. We extend old problem and propose method based on material (lattice) fixed frame of reference. It allows computing the material velocity in the reacting system in which reactions at several moving interfaces occur. All reactions lead to the lattice shift due to the difference of intrinsic diffusivities and different molar volumes. The following peculiarities are taken into account: (1) the deviation from local equilibrium at all interfaces; (2) the mobilities of the components in the bulk and interphase zone; and (3) the molar volumes of the components. We show the kinetic of the reactions, the non parabolic regime, the multiphase scale growth and present the practical application of the method. (C) 2011 American Institute of Physics. [doi:10.1063/1.3667293]

\section{INTRODUCTION}

In this work, we extend old problem of the phase competition that is usually solved based on steady-state approximation and propose evolutionary solution based on the bi-velocity method, i.e., the generalized Darken method.

The steady-state approximation was introduced by Wagner in a case of oxide scale growing on metal ${ }^{1}$ and later used for other heterogeneous reactions, ${ }^{2-4} \mathrm{Fig}$. 1 . The homogeneity range of the intermediate phases is as a rule narrow, often not known and large deviations from stoichiometric composition are seldom observed. Thus, the concentrations and molar volume remain almost constant over the whole growing $\alpha^{j}$-phase. That is why the time derivative of concentration in the mass conservation law is negligible and, consequently, the divergence of the mass flux is close to zero: $\partial c_{i}^{j} / \partial t=-\operatorname{div} J_{i}^{j} \cong 0$. The divergence being equal to zero implies that flux does not changeover the whole phase layer and depends on the time only, i.e., $\partial J_{i}^{j} / \partial x=0 \Rightarrow J_{i}^{j}=J_{i}^{j}(t)$. Such approach (method) is called steady-state approximation. This means that the product of interdiffusion coefficient and concentration gradient is nearly equal everywhere inside the phase,

$$
\tilde{D}^{j}\left(c_{i}^{j}\right) \operatorname{grad} c_{i}^{j}=\text { const }(t)
$$

It does not imply that concentration gradient is equal everywhere, e.g., the interdiffusion coefficient may vary across the

\footnotetext{
a) Author to whom correspondence should be addressed. Electronic mail: bwierzba@agh.edu.pl.
}

layer and may have sharp minimum at stoichiometric composition (as in $\mathrm{B} 2$ phases such as $\beta$-NiAl and oxides, e.g., $\mathrm{Cr}_{2} \mathrm{O}_{3}$ ). As will be shown in the following sections, Eq. (1) integrated over the composition range of the growing layer (for arbitrary time) results in the integral interdiffusion coefficient that in simple situations equals the growth rate constant of the $\alpha^{j}$-phase.

The solution based on the bi-velocity method presented in this work allows computing the material velocity in the binary reacting system in which reactions at several moving interfaces occur. All reactions lead to the lattice shift. We take into account the difference of intrinsic diffusivities and molar volumes and the deviation from the local equilibrium at all interfaces. We do not consider stresses and temperature gradients. The outline of the work is as follows:

- the steady-state approximations of the reactive diffusion and integral interdiffusion coefficient. From Wagner and Darken to the quasi-stationary bi-velocity method for the binary two phase system;

- the bi-velocity method of the multiphase growth;

- the comparison of the steady state approximation and presented method for $\mathrm{Cu}-\mathrm{Sn}$ system.

\section{INTEGRAL INTERDIFFUSIVITY}

\section{A. The Wagner method: Parabolic rate constant and Wagner integral diffusivity}

Carl Wagner formulated his model to analyze the reactive diffusion in ionic compounds, Fig. 1. Wagner assumed that: (1) the diffusion fluxes are coupled by the electric field, (2) the reactions are restricted to the two planar phase boundaries where the para-equilibrium conditions hold, ${ }^{5}$ 


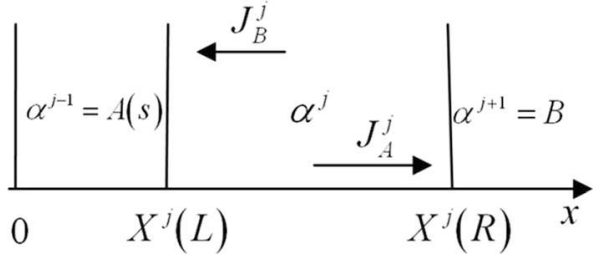

FIG. 1. The growth of the binary $\alpha^{j}$ phase between pure elements $A$ and $B$; $X^{j}(L)$ and $X^{j}(R)$ at the moving interfaces.

(3) the binary compound is formed, (4) the reaction product shows low non-stoichiometry and steady-state approximation is valid, (5) the terminal phases (substrates A and B) are the pure elements, and (6) the diffusivities vary within the narrow homogeneity range of the growing compound. Wagner method was later used to introduce the Wagner's integral diffusion coefficient (integral interdiffusivity) in a metallic binary compound in which reactive diffusion occurs.

By combining diffusion fluxes with the mass balances at interfaces (the Stefan condition at moving boundary), the velocity of interface is given by

$$
\left\langle c_{i}^{j}\right\rangle \dot{X^{j}}={ }^{d} \tilde{J}_{i}^{j}(t) \Rightarrow \dot{X^{j}}=\frac{1}{\left\langle c_{i}^{j}\right\rangle} d \tilde{J}_{i}^{j}(t)=-\tilde{D}^{j} \frac{\partial^{*} N_{i}^{j}}{\partial x},
$$

where diffusivity is expressed by the interdiffusion coefficient; ${ }^{*} N_{i}^{j}=N_{i}^{j} /\left\langle c_{i}^{j}\right\rangle$ denotes the molar ratio of $i$-component in its sublattice (because we consider compounds showing low nonstoichiometry, the average concentrations are composition invariant: $\left\langle c_{i}^{j}\right\rangle \cong$ const). Elementary transformations of Eq. (2) allow obtaining the kinetic equation by integrating the flux over the whole growing layer,

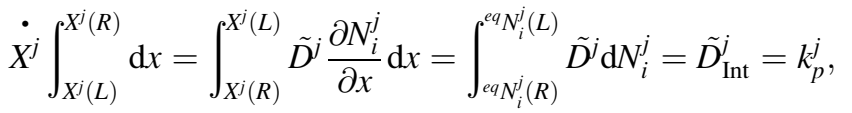

where $\int_{X^{j}(L)}^{X^{j}(R)} \mathrm{d} x=X^{j}(R)-X^{j}(L)=X^{j}(t)$ is the thickness of the layer shown in Fig. 1. We can write Eq. (3) in usual "parabolic form,"

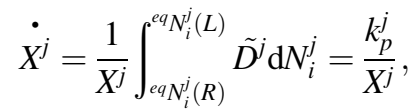

where $k_{p}^{j}=\int_{{ }^{e q} N_{i}^{j}(R)}^{e q} N^{j}(L) \tilde{D}^{j} \mathrm{~d} N_{i}^{j}=\tilde{D}_{\text {Int }}^{j}$ is the Wagner integral interdiffusivity which in the case of oxides is given by $\tilde{D}_{\text {Int }}^{j}=D_{O}+D_{M e} z_{c} /\left|z_{a}\right|^{1}$

Equations (3) and (4) show the well known fact that the rate of solid state reactions is not determined exclusively by the diffusivity and the homogeneity range but at all times by their product: the integral coefficient. The Wagner integral coefficient is used for the oxides and intermetallic compounds. Relations (2) and (3) hold when terminal phases are pure components. In multi-layer systems the rate constants differ up to orders of magnitude from integral interdiffusivities, i.e., from the value given by Eq. (3).

\section{B. The Darken method, parabolic rate constant, and Darken integral interdiffusivity}

The integral interdiffusion coefficient in many cases can be computed using Darken's method. ${ }^{6}$ When the local equilibrium exists at interfaces, then the thermodynamic factor and Darken integral interdiffusivity are given by Eq. (5) (derived in Appendix A),

$$
\begin{aligned}
\tilde{D}_{\text {Int }}^{j}= & -\left(D_{2}^{j} N_{1}^{j}+D_{1}^{j} N_{2}^{j}\right) \frac{N_{1}^{j} N_{2}^{j}}{k T \Delta^{e q} N_{2}^{j}} \\
& \times\left[\frac{g^{j+1}-g^{j}}{e q N_{2}^{j}(R)-e q N_{2}^{j+1}(L)}+\frac{-g^{j}+g^{j-1}}{e q N_{2}^{j-1}(R)-e q N_{2}^{j}(L)}\right],
\end{aligned}
$$

where $g^{j}$ denote the Gibbs potential per one atom of neighboring phases (IM compounds).

The Darken and Wagner methods are self-consistent and dominate in the reactive diffusion studies. ${ }^{1,6}$ Wagner method is still used for the diffusion controlled growth of the compounds showing two distinct sublattices and where the electric field is non-negligible, e.g., the diffusion controlled growth of the ionic compounds (oxides, sulfides, etc). The Darken method is particularly useful in case of intermetallics and compounds that can be treated as solid solutions within their narrow homogeneity range.

Remarks: In deriving Eqs. (3) and (5), the steady state growth (the parabolic regime) and local-equilibrium at both phase boundaries were assumed. Consequently, their use is restricted:

1. The integral coefficients can be evaluated from the experimental data only when the parabolic regime of the single layer growth is known.

2. All formulae are valid when local equilibrium (para or ortho-equilibrium) at interfaces exists.

3. Eq. (3) allows computing fluxes in both, the single as well as multi-layer binary systems when steady-state assumption holds and integral interdiffusivity is known. However,

4. Eq. (3) does not allow computing the reaction rate $\left(k_{p}^{j} \neq \tilde{D}_{\text {Int }}^{j}\right)$ in multi-layer systems.

Multi phase growth and non-steady processes require more general approach shown in the next sections.

\section{Two-phase growth}

The problem of "the first phase to grow," the more general problem of the sequential phase formation and related problem of the non-parabolic growth laws in solid-state reactions remain a challenge for several generations of researchers. Beside the diffusion in product layer, the existing methodologies consider additional factors determining the evolution of the binary system shown in Fig. 2:

1. the thermodynamic or kinetic suppression of some phases at nucleation stage,

2. the finite rate of mass transport through the interphase zone or, in the other words, the deviations from local equilibrium at the moving phase boundaries, 


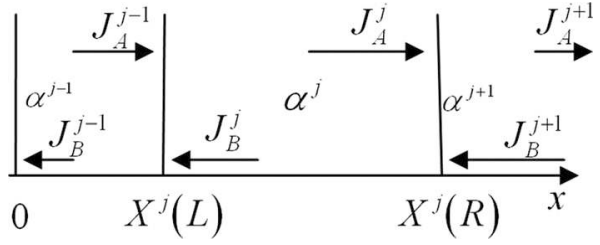

FIG. 2. The growth of the $\alpha^{j}$ phase between the two terminal binary solid solutions; $X^{j}(L)$ and $X^{j}(R)$ at the moving interfaces.

3. the effect of the fast diffusion paths,

4. the extreme principle methods, i.e., the use of semiempirical principle of maximum release rate of Gibbs free energy.

The physically clear and most widely known method from the second group (the influence of interface kinetics) was presented by Gösele and Tu already in $1982 .^{7}$ The method predicts the well known linear-parabolic growth law for the single phase growth. For the case of two intermediate phases indicates the criterion of phase suppression, offers the manner of calculation of the delay time of the suppressed phase. Gusak and Gurov approach $^{8}$ based on the diffusive competition between critical nuclei of all phases as well as between the growing phase layers. Both methods were combined and allow account of interface kinetics and nucleation in the concentration gradient. ${ }^{9}{ }^{10}$ They contain the following implicit assumptions:

1. The mass transport is a steady-state process, i.e., the fluxes of components in growing sub-layers and through their boundaries differ from phase to phase but are position invariant within each phase. Accordingly, the fluxes depend on time only ${ }^{d} J_{i}^{j}(t)$.

2. The diffusion fluxes are defined in the laboratory (Matano) reference frame. ${ }^{11}$
3. The diffusion fluxes are expressed by the first Fick's law with the assumptions of composition independent interdiffusivity and only the linear concentration profiles follow.

4. The concentrations at phase boundaries can deviate from local equilibrium at both sides of each phase boundary. The flux in the phase layer in the vicinity of phase boundary is supposed to be proportional to composition deviation only at corresponding side of this phase boundary. Deviation at the other side of the phase boundary zone is assumed to be linked only with flux across next phase layer.

The first assumption (steady-state) only seldom leads to high error. The others often lead to underestimation of the important regimes. The fourth assumption decouples the deviations of the local concentrations at the two sides of the moving phase boundary. It is apparent that, in general, the flux across phase boundary [shown as the interphase zone in Fig. (3c)] depends on conditions at both sides of the phase boundary zone and all the moving interfaces.

\section{THE QUASI-STATIONARY METHOD FOR THE BINARY TWO PHASE SYSTEM, ANALYTICAL SOLUTION}

We do consider the growth of two phases between the pure terminal components (phases $\alpha^{0}$ and $\alpha^{3}$ ), Fig. 3. We will not consider here the case of the composition dependent partial molar volumes. The partial molar volumes differ from phase to phase but otherwise are constant. In the case of phases showing narrow composition range $\left(c_{i}^{j} \cong\right.$ const), the mass conservation law reduces to the steady-state form and implies the position independent fluxes of both components,

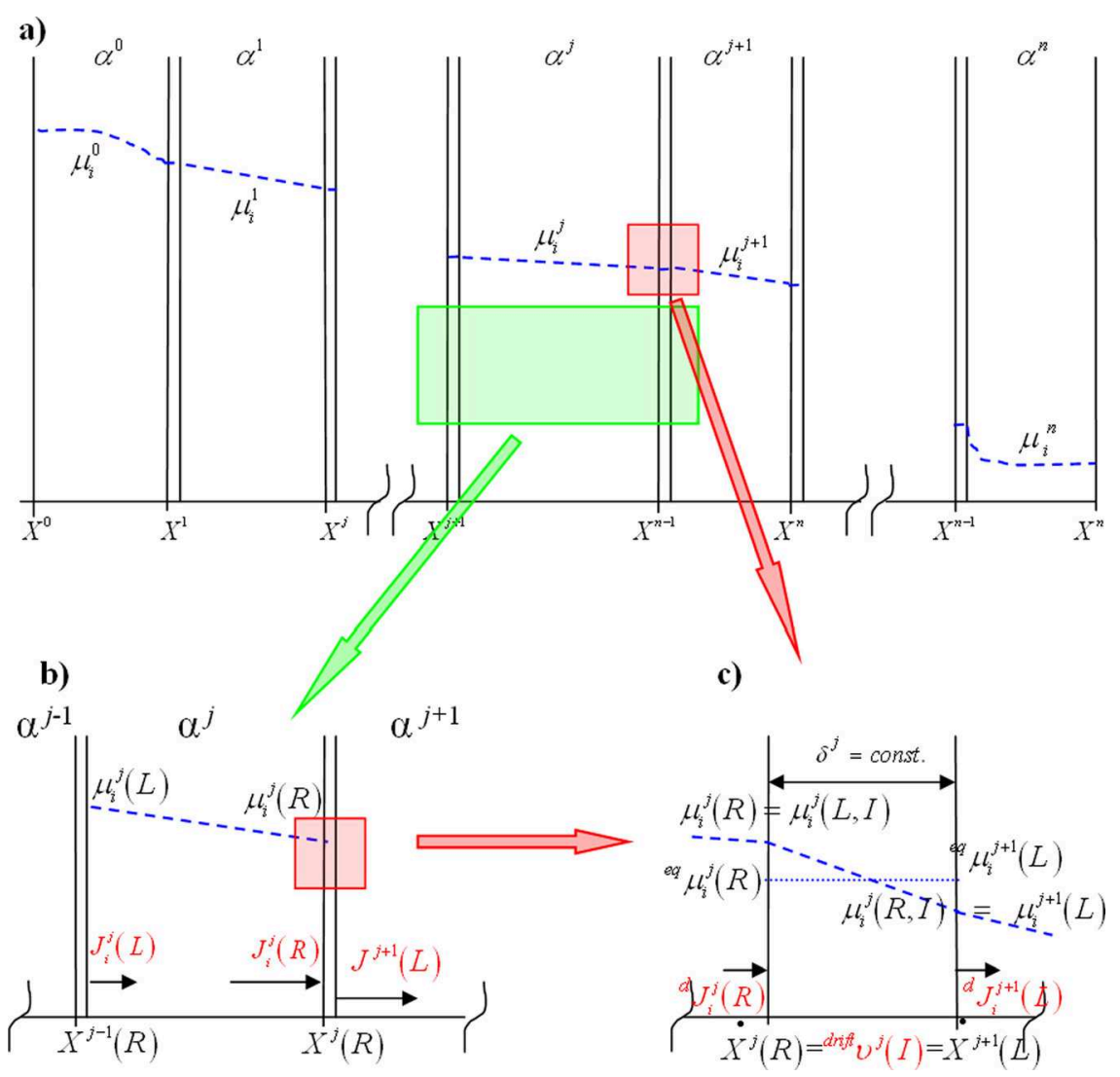

FIG. 3. (Color online) Heterogeneous solid state reaction; (a) the multiphase product layer between terminal phases $\alpha^{0}$ and $\alpha^{n}$; (b) $\alpha^{j}$ phase with left and right moving phase boundaries; (c) $\alpha^{j} \mid \alpha^{j+1}$ phase boundary zone where $X^{j+1}(L)=X^{j}(R)+\delta^{j}$ is position of the left boundary of the $\alpha^{j+1}$-phase. 


$$
\operatorname{div}\left(c_{i}^{j} v_{i}^{j}\right)=0 \Rightarrow J_{i}^{j}=c_{i}^{j} v_{i}^{j}=J_{i}^{j}(t)
$$

The $i$-component flux in every phase (and phase boundary zone) is expressed as molar, $J_{i}^{j}:=c_{i}^{j} v_{i}^{j}$, and volume, ${ }^{V} J_{i}^{j}:=c_{i}^{j} \Omega_{i}^{j} v_{i}^{j}$, flux. When Kirkendall effect inside the layer is negligible, the drift is produced at interfaces only. The velocity of the $i$-component is affected by reactions and is a sum of diffusion and common drift that is generated at interfaces of the phase boundary zone, Fig. 3 ,

$$
v_{i}^{j}={ }^{d} v_{i}^{j}+{ }^{d r i f t} v^{j} \Rightarrow J_{i}^{j}=c_{i}^{j} v_{i}^{j}=c_{i}^{j d} v_{i}^{j}+c_{i}^{j} d r i f v^{j} .
$$

Consequently, we have new variable (common drift velocity) that requires additional relation. ${ }^{12}$

Volume continuity equation (VCE): The overall and partial molar volumes, $\Omega_{i}^{j}$, are intensive parameters and are not conserved: $\Omega_{i}^{j}=\Omega_{i}^{j}\left(N_{1}, \ldots, N_{r} ; p, T\right)$. On the other hand, the overall molar volume density of the every phase equals unity,

$$
c^{j} \Omega^{j}=\sum_{i} c_{i}^{j} \Omega_{i}^{j}=1,
$$

and allows determining the volume of the evolving/reacting system. The integral form of the volume continuity equation $^{12,13}$ is given by

$$
\frac{\mathrm{d} V^{j}}{\mathrm{~d} t}=\int_{V^{j}(t)} \frac{\partial v^{j}}{\partial x} \mathrm{~d} x
$$

where $V^{j}=V^{j}(t)$ denotes the volume occupied by $j$-phase (not its molar volume, $\Omega^{j}$ ) and $v^{j}=\sum_{i} c_{i}^{j} \Omega_{i}^{j} v_{i}^{j}$ is the volume velocity in the $j$-phase.

At the constant pressure, temperature, and partial molar volumes, $\Omega_{i}^{j}=$ const. and in one-dimensional case analyzed here, the differential form of the VCE, is given by

$$
\frac{\partial}{\partial x} \sum_{i} \Omega_{i}^{j} c_{i}^{j} v_{i}^{j}=\frac{\partial}{\partial x} \sum_{i} \Omega_{i}^{j} c_{i}^{j}\left({ }^{d} v_{i}^{j}+{ }^{D} v^{j}\right)=0
$$

where the drift velocity, ${ }^{D} v^{j}={ }^{d r i f t} v^{j}$, is caused by the non balanced diffusion of components only. From Eq. (10) in each phase we have

$$
D_{v^{j}}=-\sum_{i} \Omega_{i}^{j d} J_{i}^{j} \quad \text { for } \quad j=0,1, \ldots, n .
$$

Upon introducing the proper boundary conditions, the integral form of the VCE, Eq. (9), allows computing the volume change as a result of chemical reactions at interfaces of the reacting system.

We consider now the binary system in which

1. the quasi-stationary mass transport occurs and Eq. (6) holds;

2. the stresses are negligible, the all partial molar volumes are constant and equal; and

3. the reactions and fluxes through the interfaces (boundary conditions) affect the every phase volume and volume velocity.

\section{A. Boundary conditions follow from Leibnitz's rule of calculus}

$\frac{\mathrm{d}}{\mathrm{d} t} \int_{a(t)}^{b(t)} f(x, t) \mathrm{d} x=\int_{a(t)}^{b(t)} \frac{\partial}{\partial t} f(x, t) \mathrm{d} x+\dot{b}(t) f(t, b)-\dot{a}(t) f(t, a)$.

The mass balance at moving interfaces follows from Eq. (12), $f(t, x)=c_{i}^{j}$. For $i=1, \ldots, r$ and $j=0, \ldots, n$

$$
\left(c_{i}^{j}(R)-\left\langle c_{i}^{j}(I)\right\rangle\right) \dot{X^{j}}=J_{i}^{j}(R)-{ }^{d} J_{i}^{j}(I)-\left\langle c_{i}^{j}(I)\right\rangle D v^{j}(I),
$$

$$
\left(c_{i}^{j+1}(L)-\left\langle c_{i}^{j}(I)\right\rangle\right) \dot{X^{j}}=J_{i}^{j+1}(L)-{ }^{d} J_{i}^{j}(I)-\left\langle c_{i}^{j}(I)\right\rangle D v^{j}(I),
$$

where $\dot{X^{j}}=\mathrm{d} X^{j} / \mathrm{d} t$ is the interface velocity; ${ }^{d} J_{i}^{j}(I)$ $-\left\langle c_{i}^{j}(I)\right\rangle^{D} v^{j}(I)$ denotes the overall flux within the interface boundary zone (sum of the diffusion and Darken fluxes), i.e., for $x \in\left(X^{j}(t), X^{j}(t)+\delta^{j}\right)$ and $c_{i}^{j}(R)=c_{i}^{j}\left(X^{j}(t)\right)$ denotes the $i$-component concentration at the right side of the $\alpha^{j}$ phase.

Combining Eqs. (13) and (14), the velocity of the $j$-phase right boundary can now be expressed by the fluxes in the neighboring phases,

$$
\dot{X^{j}}=\frac{\left(c_{i}^{j+1} d v_{i}^{j+1}+c_{i}^{j+1} D_{v^{j+1}}\right)(L)-\left(c_{i}^{j d} v_{i}^{j}+c_{i}^{j D} v^{j}\right)(R)}{c_{i}^{j+1}(L)-c_{i}^{j}(R)},
$$

for $i=1, \ldots, \mathrm{r}$ and $j=0, \ldots, n-1$.

Finally, by multiplying both sides of Eq. (15) by $c_{i}^{j} \Omega_{i}^{j}$ and summing overall components, Eq. (15) becomes

$$
\dot{X^{j}}=\sum_{i} c_{i}^{j} \Omega_{i}^{j} \frac{\left(c_{i}^{j+1} d v_{i}^{j+1}+c_{i}^{j+1} D_{v^{j+1}}\right)(L)-\left(c_{i}^{j d} v_{i}^{j}+c_{i}^{j D} v^{j}\right)(R)}{c_{i}^{j+1}(L)-c_{i}^{j}(R)},
$$

for $j=0, \ldots, n-1$.

The phase volume (in one-dimensional case equals the layer thickness, Fig. 3) follows from Eq. (16),

$$
\Delta X^{j}(t)=X^{j+1}-X^{j} \quad \text { for } \quad j=0, \ldots, n-1 .
$$

In every phase boundary zone, the diffusion fluxes are given by the Nernst-Planck formula, Eq. (39)

$$
\begin{aligned}
{ }^{d} J_{i}^{j}(I) & =-B_{i}^{j}(I)\left\langle c_{i}^{j}(I)\right\rangle \frac{\mu_{i}^{j+1}(L)-\mu_{i}^{j}(R)}{\delta^{j}} \\
& =-B_{i}^{j}(I)\left\langle c_{i}^{j}(I)\right\rangle \frac{\mu_{i}^{j}(R, I)-\mu_{i}^{j}(L, I)}{\delta^{j}} \text { for } j=0, \ldots, n,
\end{aligned}
$$

where $B_{i}^{j}(I)$ is the invariant $i$-component mobility in the boundary zone; $\left\langle c_{i}^{j}(I)\right\rangle$ denotes the average $i$-component concentration; $\mu_{i}^{j+1}(L)=\mu_{i}^{j+1}\left(X^{j}(t)+\delta^{j}\right)$ and $\mu_{i}^{j}(R)=\mu_{i}^{j}\left(X^{j}(t)\right)$ are time dependent chemical potentials at interfaces of the $\alpha^{j} \mid \alpha^{j+1}$ phase boundary zone and $\delta^{j}$ is the constant thickness of the $j$-phase boundary zone. 
Shift from the local equilibrium at interfaces in the binary multiphase system: To solve the evolutionary problem, we need boundary conditions allowing calculations of the concentrations of the components on each side of the phase boundary zone shown in Fig. 3(c). Upon expressing the force as a function of shift from the local equilibrium on the both sides of the interphase zone, the diffusion fluxes, $J_{i}^{j}(I)$, are given by (Appendix B),

$$
\begin{aligned}
J_{1}^{j}(I) \cong & -\frac{B_{1}^{j}(I) c_{1}^{j}(I)}{\delta^{j}}\left(\left({ }^{e q} N_{1}^{j+1}-N_{1}^{j+1}(L)\right) N_{2}^{j+1}(L) g^{\prime \prime j+1}\right. \\
& \left.-\left({ }^{e q} N_{1}^{j}-N_{1}^{j}(R)\right) N_{2}^{j}(R) g^{\prime \prime j}\right), \\
J_{2}^{j}(I) \cong & -\frac{B_{2}^{j}(I) c_{2}^{j}(I)}{\delta^{j}}\left(\left({ }^{e q} N_{2}^{j+1}-N_{2}^{j+1}(L)\right) N_{1}^{j+1}(L) g^{\prime \prime j+1}\right. \\
& \left.-\left({ }^{e q} N_{2}^{j}-N_{2}^{j}(R)\right) N_{1}^{j}(R) g^{\prime \prime j}\right), \quad \text { for } \quad j=0,1 \ldots, n .
\end{aligned}
$$

\section{B. The solution of the quasi-stationary method}

The position invariant volume velocity within the growing phase is generated by reactions at interfaces and follows from Eqs. (13), (14), and (39),

$$
\begin{aligned}
v^{j}= & \sum_{i} \Omega_{i}^{j} J_{i}^{j}(R)=\dot{X^{j}}+\sum_{i} \Omega_{i}^{j} J_{i}^{j}(I), \\
v^{j}= & \sum_{i} \Omega_{i}^{j} J_{i}^{j}(L)=\dot{X^{j-1}} \\
& +\sum_{i} \Omega_{i}^{j} J_{i}^{j-1}(I) \quad \text { for } \quad i=1,2 \text { and } j=0,1, \ldots, n .
\end{aligned}
$$

Introducing Eq. (20) into Eq. (9) allows computing the molar fluxes in all layers at their interfaces,

$$
\begin{aligned}
& J_{i}^{j}(R)=c_{i}^{j}(R) v^{j}-c_{i}^{j}(R) \sum_{i} \Omega_{i}^{j} J_{i}^{j}(I)+J_{i}^{j}(I), \\
& J_{i}^{j}(L)=c_{i}^{j}(L) v^{j}-c_{i}^{j}(L) \sum_{i} \Omega_{i}^{j} J_{i}^{j-1}(I)+J_{i}^{j-1}(I) \\
& \text { for } \quad i=1,2 \text { and } j=0,1, \ldots, n .
\end{aligned}
$$

In the binary mixture, $j=1,2$, the above fluxes become

$$
\begin{aligned}
& J_{2}^{j}(R)=c_{2}^{j}(R) v^{j}+\left(1-\Omega_{2}^{j} c_{2}^{j}(R)\right) J_{2}^{j}(I)-c_{2}^{j}(R) \Omega_{1}^{j} J_{1}^{j}(I), \\
& J_{2}^{j}(L)=c_{2}^{j}(L) v^{j}+\left(1-\Omega_{2}^{j} c_{2}^{j}(L)\right) J_{2}^{j-1}(I)-c_{2}^{j}(L) \Omega_{1}^{j} J_{1}^{j-1}(I),
\end{aligned}
$$

and by applying the relation $c_{1}^{j} \Omega_{1}^{j}+c_{2}^{j} \Omega_{2}^{j}=1$ we get

$$
\begin{aligned}
& J_{2}^{j}(R)=c_{2}^{j}(R) v^{j}+\Omega_{1}^{j} c_{1}^{j}(R) J_{2}^{j}(I)-\Omega_{1}^{j} c_{2}^{j}(R) J_{1}^{j}(I), \\
& J_{2}^{j}(L)=c_{2}^{j}(L) v^{j}+\Omega_{1}^{j} c_{1}^{j}(L) J_{2}^{j-1}(I)-\Omega_{1}^{j} c_{2}^{j}(L) J_{1}^{j-1}(I) .
\end{aligned}
$$

Upon expressing fluxes within the boundary by Eq. (18), one gets the analytical formulae,

$$
\begin{aligned}
J_{2}^{j}(R)= & c_{2}^{j}(R) v^{j}-\frac{\Omega_{1}^{j} c_{1}^{j}(R) B_{2}^{j}(I)\left\langle c_{2}^{j}(I)\right\rangle}{\delta^{j}}\left(\mu_{2}^{j+1}(t, L)-\mu_{2}^{j}(t, R)\right) \\
& +\frac{\Omega_{1}^{j} c_{2}^{j}(R) B_{1}^{j}(I)\left\langle c_{1}^{j}(I)\right\rangle}{\delta^{j}}\left[\mu_{1}^{j+1}(t, L)-\mu_{1}^{j}(t, R)\right], \quad \text { (24) }
\end{aligned}
$$

$$
\begin{aligned}
J_{2}^{j}(L)= & c_{2}^{j}(L) v^{j}-\frac{\Omega_{1}^{j} c_{1}^{j}(L) B_{2}^{j-1}(I)\left\langle c_{2}^{j-1}(I)\right\rangle}{\delta^{j-1}}\left(\mu_{2}^{j}(t, L)-\mu_{2}^{j-1}(t, R)\right) \\
& +\frac{\Omega_{1}^{j} c_{2}^{j}(L) B_{1}^{j-1}(I)\left\langle c_{1}^{j-1}(I)\right\rangle}{\delta^{j-1}}\left(\mu_{1}^{j}(t, L)-\mu_{1}^{j-1}(t, R)\right),
\end{aligned}
$$

where $\mu_{2}^{j}(t, R)=\mu_{2}^{j}\left(t, X^{j}\right)$ and $\mu_{2}^{j+1}(t, L)=\mu_{2}^{j+1}\left(t, X^{j}+\delta^{j}\right)$ are the steady state (time dependent) chemical potentials at both sides of $j$-phase boundary. The time dependent difference between the chemical potential at equilibrium and during the quasistationary movement of the boundary we call the local deviation of the chemical potential (from its equilibrium value).

Linear approximation for the local deviation of chemical potential: We restrict further analysis to small deviations of the chemical potentials at the boundaries (Appendix C). Using Eqs. (C5) and (C6), we express the fluxes in Eq. (22) by their deviations from the LE value. Consequently, fluxes are given by

$$
\begin{gathered}
J_{1}^{j}(R)=c_{1}^{j}(R) v^{j}-\Omega_{2}^{j} \frac{c_{1}^{j}(R) B_{2}^{j}(I)\left\langle c_{2}^{j}(I)\right\rangle N_{1}^{j}+c_{2}^{j}(R) B_{1}^{j}(I)\left\langle c_{1}^{j}(I)\right\rangle N_{2}^{j}}{\delta^{j}} \mathrm{~d} g^{\prime j}(t, R) \\
+\Omega_{2}^{j} \frac{c_{1}^{j}(R) B_{2}^{j}(I)\left\langle c_{2}^{j}(I)\right\rangle N_{1}^{j+1}+c_{2}^{j}(R) B_{1}^{j}(I)\left\langle c_{1}^{j}(I)\right\rangle N_{2}^{j+1}}{\delta^{j}} \mathrm{~d} g^{j+1}(t, L), \\
J_{2}^{j}(R)=c_{2}^{j}(R) v^{j}(t)+\frac{\Omega_{1}^{j} c_{1}^{j}(R) B_{2}^{j}(I)\left\langle c_{2}^{j}(I)\right\rangle N_{1}^{j}(R)}{\delta^{j}} \mathrm{~d} g^{\prime j}(t, R)+\frac{\Omega_{1}^{j} c_{2}^{j}(R) B_{1}^{j}(I)\left\langle c_{1}^{j}(I)\right\rangle N_{2}^{j}(R)}{\delta^{j}} \mathrm{~d} g^{\prime j}(t, R) \\
-\frac{\Omega_{1}^{j} c_{1}^{j}(R) B_{2}^{j}(I)\left\langle c_{2}^{j}(I)\right\rangle N_{1}^{j+1}(L)}{\delta^{j}} \mathrm{~d} g^{\prime j+1}(t, L)-\frac{\Omega_{1}^{j} c_{2}^{j}(R) B_{1}^{j}(I)\left\langle c_{1}^{j}(I)\right\rangle N_{2}^{j+1}(L)}{\delta^{j}} \mathrm{~d} g^{j j+1}(t, L),
\end{gathered}
$$




$$
\begin{aligned}
J_{2}^{j}(L)= & c_{2}^{j}(L) v^{j}+\frac{\Omega_{1}^{j} c_{1}^{j}(L) B_{2}^{j-1}(I)\left\langle c_{2}^{j-1}(I)\right\rangle N_{1}^{j-1}}{\delta^{j-1}} \mathrm{~d} g^{j-1}+\frac{\Omega_{1}^{j} c_{2}^{j}(L) B_{1}^{j-1}(I)\left\langle c_{1}^{j-1}(I)\right\rangle N_{2}^{j-1}}{\delta^{j-1}} \mathrm{~d} g^{\prime j-1} \\
& -\frac{\Omega_{1}^{j} c_{2}^{j}(L) B_{1}^{j-1}(I)\left\langle c_{1}^{j-1}(I)\right\rangle N_{2}^{j}}{\delta^{j-1}} \mathrm{~d} g^{\prime j}-\frac{\Omega_{1}^{j} c_{1}^{j}(L) B_{2}^{j-1}(I)\left\langle c_{2}^{j-1}(I)\right\rangle N_{1}^{j}}{\delta^{j-1}} \mathrm{~d} g^{\prime j} .
\end{aligned}
$$

Asumming negligible changes of composition in the growing phases one can further reduce above expressions for fluxes. The equal partial molar volumes in $j$-phase $\left(\Omega^{j}=\Omega_{1}^{j}=\Omega_{2}^{j}\right)$ imply $N_{1}^{j}=c_{1}^{j} \Omega^{j}=c_{1}^{j} \Omega_{1}^{j}=c_{1}^{j} \Omega_{2}^{j}$, we get

$$
\begin{aligned}
J_{1}^{j}(R)= & c_{1}^{j}(R) v^{j}-\frac{N_{1}^{j} N_{1}^{j} B_{2}^{j}(I)\left\langle c_{2}^{j}(I)\right\rangle}{\delta^{j}} \mathrm{~d} g^{\prime j}(t, R)-\frac{N_{2}^{j} N_{2}^{j} B_{1}^{j}(I)\left\langle c_{1}^{j}(I)\right\rangle}{\delta^{j}} \mathrm{~d} g^{\prime j}(t, R) \\
& +\frac{N_{1}^{j} N_{1}^{j+1} B_{2}^{j}(I)\left\langle c_{2}^{j}(I)\right\rangle}{\delta^{j}} \mathrm{~d} g^{\prime j+1}(t, L)+\frac{N_{2}^{j} N_{2}^{j+1} B_{1}^{j}(I)\left\langle c_{1}^{j}(I)\right\rangle}{\delta^{j}} \mathrm{~d} g^{j j+1}(t, L), \\
J_{2}^{j}(R)= & c_{2}^{j}(R) v^{j}(t)+\frac{N_{1}^{j} B_{2}^{j}(I)\left\langle c_{2}^{j}(I)\right\rangle N_{1}^{j}}{\delta^{j}} \mathrm{~d} g^{\prime j}(t, R)+\frac{N_{2}^{j} B_{1}^{j}(I)\left\langle c_{1}^{j}(I)\right\rangle N_{2}^{j}}{\delta^{j}} \mathrm{~d} g^{\prime j}(t, R) \\
& -\frac{N_{1}^{j} B_{2}^{j}(I)\left\langle c_{2}^{j}(I)\right\rangle N_{1}^{j+1}}{\delta^{j}} \mathrm{~d} g^{\prime j+1}(t, L)-\frac{N_{2}^{j} B_{1}^{j}(I)\left\langle c_{1}^{j}(I)\right\rangle N_{2}^{j+1}}{\delta^{j}} \mathrm{~d} g^{j j+1}(t, L), \\
J_{2}^{j}(L)= & c_{2}^{j}(L) v^{j}+\frac{N_{1}^{j} N_{1}^{j-1} B_{2}^{j-1}(I)\left\langle c_{2}^{j-1}(I)\right\rangle}{\delta^{j-1}} \mathrm{~d} g^{\prime j-1}+\frac{N_{2}^{j} N_{2}^{j-1} B_{1}^{j-1}(I)\left\langle c_{1}^{j-1}(I)\right\rangle}{\delta^{j-1}} \mathrm{~d} g^{\prime j-1}+ \\
- & \frac{N_{2}^{j} N_{2}^{j} B_{1}^{j-1}(I)\left\langle c_{1}^{j-1}(I)\right\rangle}{\delta^{j-1}} \mathrm{~d} g^{\prime j}-\frac{N_{1}^{j} N_{1}^{j} B_{2}^{j-1}(I)\left\langle c_{2}^{j-1}(I)\right\rangle}{\delta^{j-1}} \mathrm{~d} g^{\prime j} .
\end{aligned}
$$

We compress Eqs. (29)-(31) by introducing constant functions $f^{j}(R)$ and $h^{j}(R)$,

$$
\begin{array}{r}
J_{1}^{j}(R)=c_{1}^{j} v^{j}-\left[h f_{R}^{j} \mathrm{~d} g^{\prime j}(t, R)-f_{R}^{j} \mathrm{~d} g^{\prime j+1}(t, L)\right], \\
J_{2}^{j}(R)=c_{2}^{j} v^{j}+h_{R}^{j} \mathrm{~d} g^{j j}(t, R)-f_{R}^{j} \mathrm{~d} g^{j j+1}(t, L), \\
J_{2}^{j}(L)=c_{2}^{j} v^{j}+h_{L}^{j} \mathrm{~d} g^{j j-1}(t, R)-f_{L}^{j} \mathrm{~d} g^{\prime j}(t, L),
\end{array}
$$

where $f$ and $h$ are constant functions characterizing the interfaces of $j$-phase,

$$
\begin{gathered}
f_{R}^{j}=\frac{N_{1}^{j} N_{1}^{j+1} B_{2}^{j}(I)\left\langle c_{2}^{j}(I)\right\rangle+N_{2}^{j} N_{2}^{j+1} B_{1}^{j}(I)\left\langle c_{1}^{j}(I)\right\rangle}{\delta^{j}}, \\
h_{R}^{j}=\frac{N_{1}^{j} N_{1}^{j} B_{2}^{j}(I)\left\langle c_{2}^{j}(I)\right\rangle+N_{2}^{j} N_{2}^{j} B_{1}^{j}(I)\left\langle c_{1}^{j}(I)\right\rangle}{\delta^{j}} \\
h_{L}^{j}=\frac{N_{1}^{j-1} N_{1}^{j} B_{2}^{j-1}(I)\left\langle c_{2}^{j-1}(I)\right\rangle+N_{2}^{j-1} N_{2}^{j} B_{1}^{j-1}(I)\left\langle c_{1}^{j-1}(I)\right\rangle}{\delta^{j-1}}, \\
f_{L}^{j}=\frac{N_{1}^{j} N_{1}^{j} B_{2}^{j-1}(I)\left\langle c_{2}^{j-1}(I)\right\rangle+N_{2}^{j} N_{2}^{j} B_{1}^{j-1}(I)\left\langle c_{1}^{j-1}(I)\right\rangle}{\delta^{j-1}} .
\end{gathered}
$$

The example of the binary two phase analytical solution is shown in Appendix D. The final linear system of analytical equations describing the growth 2-phases between the pure terminal components is given by

$$
\begin{gathered}
-\frac{\tilde{D}^{1} \Delta^{e q} c_{2}^{1}}{X^{j}(t)}-\frac{\tilde{D}^{1} c^{1}}{X^{j}(t)}\left(\mathrm{d} N_{2}^{1}(R)-\mathrm{d} N_{2}^{1}(L)\right) \\
=-f_{L}^{1} g^{\prime \prime 1} \mathrm{~d} N_{2}^{1}(t, L), \\
-f_{L}^{1} g^{\prime \prime 1} \mathrm{~d} N_{2}^{1}(t, L) \\
=h_{R}^{1} g^{\prime \prime 1} \mathrm{~d} N_{2}^{1}(t, R)-f_{R}^{1} g^{\prime \prime 2} \mathrm{~d} N_{2}^{2}(t, L), \\
h_{L}^{2} g^{\prime \prime 1} \mathrm{~d} N_{2}^{1}(t, R)-f_{L}^{2} g^{\prime \prime 2} \mathrm{~d} N_{2}^{2}(t, L)=h_{R}^{2} g^{\prime \prime 2} \mathrm{~d} N_{2}^{2}(t, R), \\
h_{R}^{2} g^{\prime \prime 2} \mathrm{~d} N_{2}^{2}(t, R)=-\frac{\tilde{D^{2}} \Delta^{e q} c_{2}^{2}}{X^{2}(t)}-\frac{\tilde{D^{2}} c^{2}}{X^{2}(t)}\left(\mathrm{d} N_{2}^{2}(R)-\mathrm{d} N_{2}^{2}(L)\right) .
\end{gathered}
$$

Upon introducing the appropriate restrictions, the above model results in Gösele and $\mathrm{Tu}^{7}$ approximation. In the next sections, we shall compare the results of evolutionary and analytical solutions with experimental results.

\section{BI-VELOCITY METHOD; GENERALIZATION OF THE BINARY 2-PHASE SYSTEM}

The bi-velocity method allows relaxing all Gösele and Tu restrictions (the assumptions 1-4), i.e., rules out all above constraints. In the following, we show that:

1. The flux across any phase explicitly depends not only on the transport in this very phase layer but as well on the transport in the all sub-layers and through the all phase boundaries.

2. The method allows the mixed local equilibrium conditions (ortho- and/or para-equilibrium conditions ${ }^{5}$ ) at every 
a)

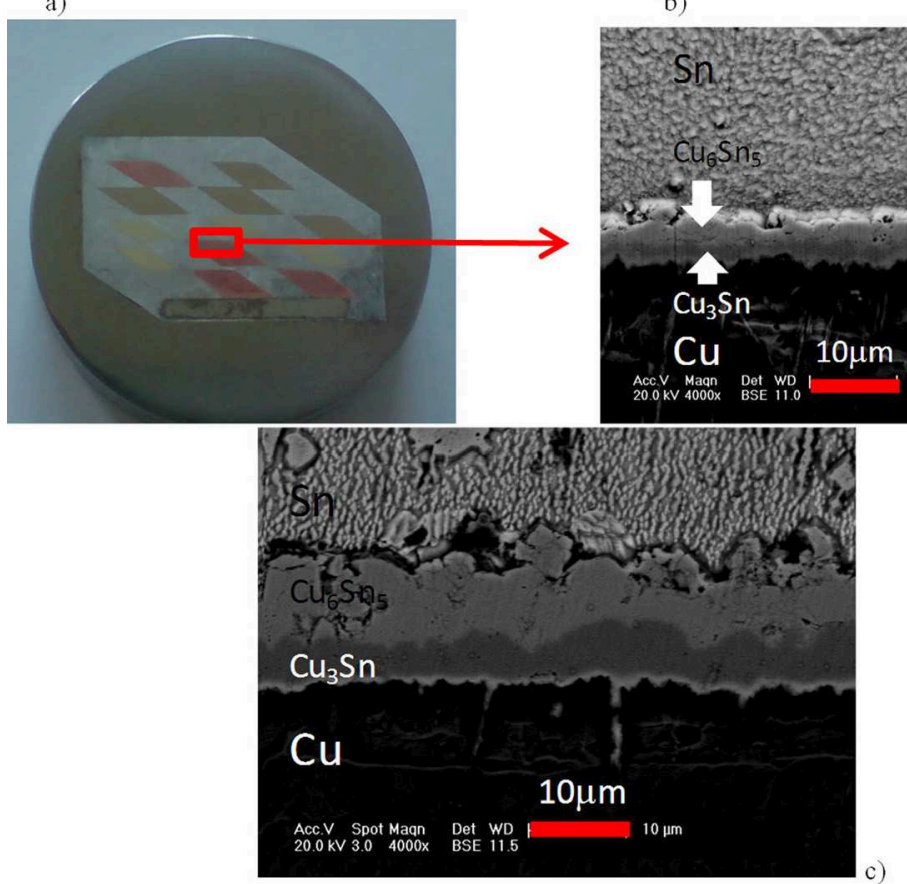

b)

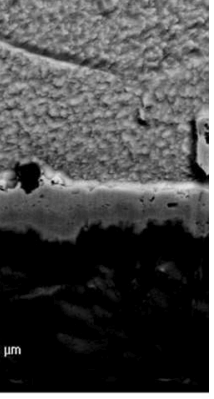

FIG. 4. (Color online) Cross sectional images of the $\mathrm{Cu}-\mathrm{Sn}-\mathrm{Ag}-\mathrm{Ni}$ diffusion multiple: (a) overall view; (b) BSE cross section images of the $\mathrm{Cu}-\mathrm{Sn}$ zone after HIP process at $210^{\circ} \mathrm{C}$; (c) and after annealing at $200^{\circ} \mathrm{C}$ for $336 \mathrm{~h}$.

phase boundary, i.e., on both sides of each phase boundary zone shown in Fig. 3(c).

3. The non-steady-state reactive diffusion in $\mathrm{Cu}-\mathrm{Sn}$ system can be effectively simulated using the bi-velocity method.

4. The steady state and evolutionary solutions converge when reactive diffusion in $\mathrm{Cu}-\mathrm{Sn}$ system is simulated. It confirms the self-consistency of the quantitative analytical formulae describing the steady-state reactive diffusion and the nonsteady-state approach based on the bi-velocity method.

We analyze the binary, multiphase reacting system, shown in Fig. 3, with emphasis on the evolution of the deviation from the local equilibrium at phase boundaries. As a result of the shift from the local equilibrium caused by the transport through the interface zone, the diffusion potentials differ from the time independent equilibrium values, ${ }^{e q} \mu_{i}^{j}(R)$ and ${ }^{e q} \mu_{i}^{j+1}(L)$. The key concept introduced by us is the two-sided shift from the local equilibrium at every interface. Namely, we consider the mass transport through the phase boundary zone and assume ortho-equilibrium at both interfaces of each phase boundary zone shown in
Fig. 3(c). We postulate that time dependent diffusion potentials at interfaces obey the following equalities:

$$
\mu_{i}^{j}(R)=\mu_{i}^{j}(L, I) \quad \text { and } \quad \mu_{i}^{j+1}(L)=\mu_{i}^{j}(R, I) .
$$

We consider a noncompensated diffusion fluxes that can generate drift at interfaces (due to chemical reaction-Wagner method) as well as within the diffusion zone (Kirkendall effect). ${ }^{13}$ In the binary multiphase system the bi-velocity method means: ${ }^{13,24}$

1. The fluxes of components in every phase and phase boundary zone are expressed as molar and volume flux: $J_{i}^{j}:=c_{i}^{j} v_{i}^{j}$ and ${ }^{V} J_{i}^{j}:=c_{i}^{j} \Omega_{i}^{j} v_{i}^{j}$, respectively.

2. The velocity of the component is affected by reactions and is a sum of diffusion and common drift generated at interfaces and/or within the growing phase (Kirkendall effect).

$$
v_{i}^{j}={ }^{d} v_{i}^{j}+{ }^{d r i f t} v^{j}
$$

TABLE I. Partial molar volumes of components $\left[\mathrm{cm}^{3} \mathrm{~mol}^{-1}\right]$, standard Gibbs free energy per atom $\left[\mathrm{kg} \mathrm{cm}^{2} \mathrm{~s}^{-2}\right]$ and its derivatives in the Cu-Sn system at 473 K (Refs. 24 and 25).

\begin{tabular}{lccc}
\hline \hline Phase, $j$ & $\mathrm{Cu}$ & $\mathrm{Cu}_{3} \mathrm{Sn}-\varepsilon$ & $\mathrm{Cu}_{6} \mathrm{Sn}_{5}-\eta$ \\
\hline$j$ & 0 & 1 & 2 \\
$\Omega_{1}^{j}=\Omega_{2}^{j}$ & 7.36 & 6.90 & 11.39 \\
$g_{j}={ }^{o} G^{j}$ & $-2.785 \cdot 10^{-16}$ & $-4.521 \cdot 10^{-16}$ & $-4.592 \cdot 10^{-16}$ \\
$\Delta N_{S n}$ & 0.005 & 0.01 & -16.28 \\
$g^{\prime j}(L)=\frac{g^{j}-g^{j-1}}{N_{S n}^{j}(L)}$ & $-7.08 \cdot 10^{-16}$ & $-1.61 \cdot 10^{-17}$ \\
$g^{\prime j}(R)=-\frac{g^{j}-g^{j+1}}{N_{C u}^{j}(R)}$ & $-9.47 \cdot 10^{-18}$ & $6.73 \cdot 10^{-17}$ \\
$g^{\prime \prime j}=\frac{g^{\prime j}(R)-g^{\prime j}(L)}{a}$ & & $1.39 \cdot 10^{-13}$ & $8.34 \cdot 10^{-15}$ \\
\hline \hline
\end{tabular}


TABLE II. The $\mathrm{Cu}$ and $\mathrm{Sn}$ mobilities and interdiffusion coefficients in different phases and interfaces at $473 \mathrm{~K}$ (Refs. 19, 26 and 27).

\begin{tabular}{|c|c|c|c|}
\hline Phase and its cipher, $\alpha^{j}$ & & $B_{\mathrm{Sn}, \mathrm{Cu}}^{j}, s / k g$ & $\begin{array}{l}\text { Interdiffussion } \\
\text { coefficient, } \mathrm{cm}^{2} / \mathrm{s}\end{array}$ \\
\hline $\mathrm{Cu} \mid \mathrm{Cu}_{3} \mathrm{Sn}$ & & $1.68 \cdot 10^{5}$ & $1.1 \cdot 10^{-11}$ \\
\hline $\mathrm{Cu}_{3} \mathrm{Sn}$ & 2 & $1.68 \cdot 10^{5}$ & $1.1 \cdot 10^{-11}$ \\
\hline $\mathrm{Cu}_{3} \mathrm{Sn} \mid \mathrm{Cu}_{6} \mathrm{Sn}_{5}$ & & $1.75 \cdot 10^{5}$ & $1.15 \cdot 10^{-11}$ \\
\hline $\mathrm{Cu}_{6} \mathrm{Sn}_{5}$ & 3 & $1.84 \cdot 10^{5}$ & $1.2 \cdot 10^{-11}$ \\
\hline $\mathrm{Cu}_{6} \mathrm{Sn}_{5} \mid \mathrm{Sn}$ & & $1.84 \cdot 10^{5}$ & $1.2 \cdot 10^{-11}$ \\
\hline
\end{tabular}

3. The Nernst-Planck flux is the constitutive formula for diffusion velocity,

${ }^{d} v_{i}^{j}=-B_{i}^{j} \operatorname{grad} \mu_{i}^{j}=-B_{i}^{j} \frac{\partial \mu_{i}^{j}}{\partial N_{i}^{j}} \operatorname{grad} N_{i}^{j}=-B_{i}^{j} \frac{\partial^{2} g^{j}}{\partial\left(N_{i}^{j}\right)^{2}} \operatorname{grad} N_{i}^{j}$.

The composition dependence of the chemical potential might be a known function or approximation. Thus, the diffusion fluxes are given by

$$
\begin{aligned}
& { }^{d} J_{1}^{j}=-B_{1}^{j} c_{1}^{j} \operatorname{grad} \mu_{1}^{j}=-B_{1}^{j} c_{1}^{j} N_{2}^{j} g^{\prime \prime j} \operatorname{grad} N_{1}^{j}, \\
& { }^{d} J_{2}^{j}=-B_{2}^{j} c_{2}^{j} \operatorname{grad} \mu_{2}^{j}=-B_{2}^{j} c_{2}^{j} N_{1}^{j} g^{\prime \prime j} \operatorname{grad} N_{2}^{j} .
\end{aligned}
$$

In this work, we use the linear approximation of Gibbs free energy. In the Appendix A, we show examples of such linear approximation, $g^{\prime \prime j}=\partial^{2} g^{j} / \partial\left(N_{1}^{j}\right)^{2}=\partial^{2} g^{j} / \partial\left(N_{2}^{j}\right)^{2}$.

\section{A. Laws, boundary, and initial conditions}

\section{The mass conservation law}

We do not consider the chemical reactions within the diffusion zone (reactions occur at interfaces only) and the mass continuity equation is given by

$$
\frac{\partial c_{i}^{j}}{\partial t}+\operatorname{div}\left(c_{i}^{j} v_{i}^{j}\right)=0 \text { for } i=1,2 \text { and } j=0, \ldots, n,
$$

the concentrations, $c_{i}^{j}$, and velocities of components, $v_{i}^{j}$, depend on time and position.

The overall component velocity in the system shown in Fig. 3 equals $v_{i}^{j}={ }^{d} v_{i}^{j}+{ }^{d r i f t} v^{j}$, where drift is given by
${ }^{d r i f t} v^{j}={ }^{D} v^{j}+{ }^{t r} v^{j}(t)$, i.e., the drift is due to the reactions at proceeding interfaces, ${ }^{t r} v^{j}(t)=v^{j-1}(I)$, and Kirkendall effect, ${ }^{D} v^{j}$. The flux relatively to left boundary of the $j$-phase, $X^{j}(L, t)=X^{j-1}(R, t)+\delta^{j-1}, \quad$ can be expressed by $v_{i}^{j}={ }^{d} v_{i}^{j}+{ }^{D} v^{j}$. Thus, the mass conservation in the $j$-phase is given by

$$
\begin{aligned}
& \frac{\partial c_{i}^{j}}{\partial t}+\operatorname{div}\left(c_{i}^{j d} v_{i}^{j}\right)+\operatorname{div}\left(c_{i}^{j D} v^{j}\right)=0 \\
& \text { for } i=1,2 \text { and } j=0, \ldots, n,
\end{aligned}
$$

where ${ }^{d} J_{i}^{j}=c_{i}^{j d} v_{i}^{j}$ is the diffusion flux and ${ }^{D} v^{j}$ denotes the Darken velocity given by Eq. (11).

Volume continuity equation and boundary conditions were already presented in Sec. III.

\section{Initial conditions}

1. The known initial distributions of the components in every phase,

$$
c_{i}^{j}(0, x)=\stackrel{\circ}{c}_{i}^{j}(x) \quad \text { for } \quad i=1,2 \quad \text { and } \quad j=0, \ldots, n \text {. }
$$

2. The initial thickness of every sublayer ( $j$-phase) $\Delta X^{j}(t=0)=X_{0}^{j}(R)-X_{0}^{j}(L)$ for $j=0, \ldots, n$.

3. Constant thickness of the every phase boundary zone, $\delta^{j}$.

\section{B. The numerical solution}

\section{Initial boundary value problem of multiphase binary system}

\section{Data:}

(a) The partial molar volumes of the components in each phase, $\Omega_{i}^{j}$;

(b) The diffusion coefficient of the i-th component in $j$-th phase, $D_{i}^{j}$

(c) The second derivative of the Gibbs potential in $j$-th phase, $g^{\prime \prime j}$

(d) Equilibrium molar fraction of the i-th component in $j$-th phase, ${ }^{e q} N_{i}^{j}$;

(e) Duration of the process, $t$;

(f) The constant process temperature, $T$;

(g) Constant thickness of the every phase boundary zone, $\delta^{j}$.
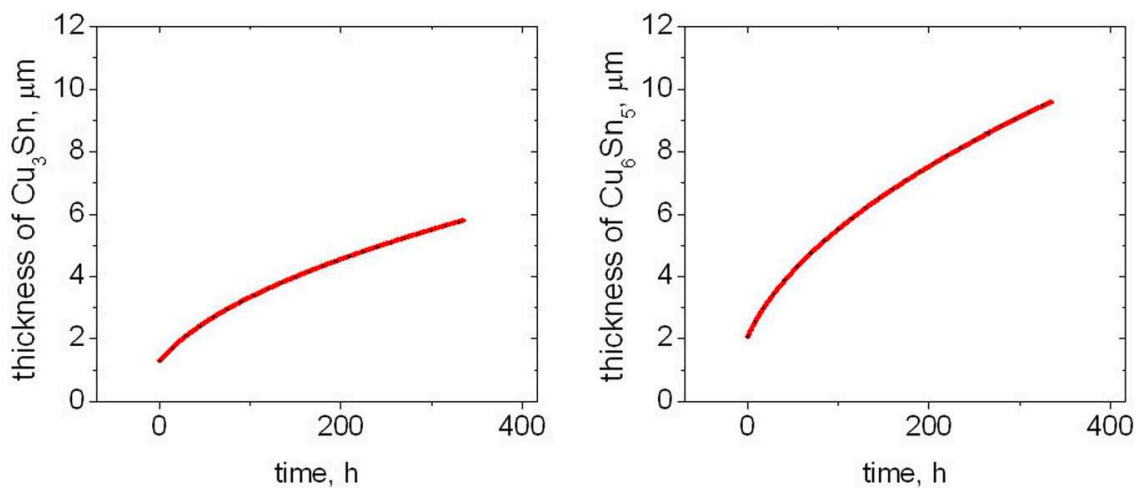

FIG. 5. (Color online) The numerical simulation of the diffusion controlled simultaneous growth of the intermetallic compounds between pure $\mathrm{Cu}$ and $\mathrm{Sn}$ at $473 \mathrm{~K}$. The analytical steady-state and the evolutionary solutions converge. 
a)

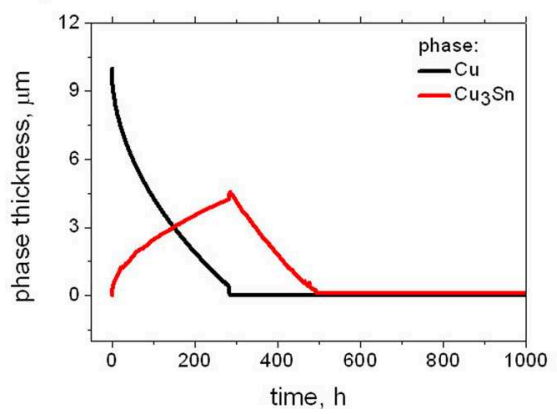

b)

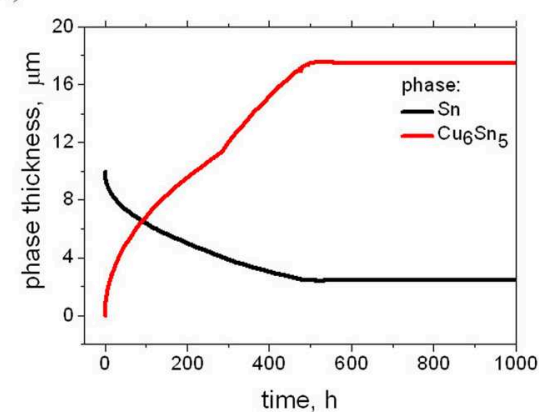

FIG. 6. (Color online) The time evolution of the finite $\mathrm{Cu}-\mathrm{Sn}$ diffusion couple. The thickness of the (a) $\mathrm{Cu}, \mathrm{Cu}_{3} \mathrm{Sn}$ and (b) $\mathrm{Sn}, \mathrm{Cu}_{6} \mathrm{Sn}_{5}$ layers as a function of time during the elongated reaction period.

The unknowns:

Laws:

a) Concentration of the i-th component in j-th phase, $c_{i}^{j}$;

b) The position of the phase boundaries, $X^{j}$.

\section{Initial conditions:}

a) The known initial distributions of the components in every phase, $c_{i}^{j}(0, x)=\stackrel{\circ}{c}_{i}^{j}(x)$;

b) The initial position of the phase boundaries, $X^{j}(t=0)$. a) $\frac{\partial c_{i}^{j}}{\partial t}+\operatorname{div}\left(c_{i}^{j} d v_{i}^{j}\right)+\operatorname{div}\left(c_{i}^{j} D_{v^{j}}\right)=0$ for $i=1, \ldots, r$ and
$j=0, \ldots, n$,

b) $D_{v^{j}}=-\sum_{i} \Omega_{i}^{j} c_{i}^{j d} v_{i}^{j}$ for $j=0,1, \ldots, n$,

c) ${ }^{d} v_{i}^{j}=-B_{i}^{j} \operatorname{grad} \mu_{i}^{j}$ for $i=1, \ldots, r$ and $j=0, \ldots, n$.

\section{Boundary conditions:}

(a) $\left.\left(c_{i}^{j}(R)-\left\langle c_{i}^{j}(I)\right\rangle\right) \dot{X}^{j}=J_{i}^{j}(R)-{ }^{d} J_{i}^{j}\right)-\left\langle c_{i}^{j}(I)\right\rangle{ }^{D} v^{j}(I)$, $\left(c_{i}^{j+1}(L)-\left\langle c_{i}^{j}(I)\right\rangle\right) \dot{X}^{j}=J_{i}^{j+1}(L)-{ }^{d} J_{i}^{j}(I)-\left\langle c_{i}^{j}(I)\right\rangle^{D} v^{j}(I)$,

(b) $\dot{X^{j}}=\sum_{i} c_{i}^{j} \Omega_{i}^{j} \frac{\left(c_{i}^{j+1} \Omega_{i}^{j+1} v_{v_{i}^{j+1}}+c_{i}^{j+1} \Omega_{i}^{j+1} D_{v^{j+1}}\right)(L)-\left(c_{i}^{j} \Omega_{i}^{j} v_{i}^{j}+c_{i}^{j} \Omega_{i}^{j} D_{v^{j}}\right)(R)}{c_{i}^{j+1} \Omega_{i}^{j+1}(L)-c_{i}^{j} \Omega_{i}^{j}(R)}$,

(c) $\quad J_{1}^{j}(I) \cong-\frac{B_{1}^{j}(I) c_{1}^{j}(I)}{\delta^{j}}\left(\left({ }^{e q} N_{1}^{j+1}-N_{1}^{j+1}(L)\right) N_{2}^{j+1}(L) g^{\prime \prime j+1}-\left({ }^{e q} N_{1}^{j}-N_{1}^{j}(R)\right) N_{2}^{j}(R) g^{\prime \prime j}\right)$,

$$
J_{2}^{j}(I) \cong-\frac{B_{2}^{j}(I) c_{2}^{j}(I)}{\delta^{j}}\left(\left(e q N_{2}^{j+1}-N_{2}^{j+1}(L)\right) N_{1}^{j+1}(L) g^{\prime \prime j+1}-\left({ }^{e q} N_{2}^{j}-N_{2}^{j}(R)\right) N_{1}^{j}(R) g^{\prime \prime j}\right) .
$$

The above set of differential equations was numerically solved in following steps: (i) the discretization part (reformulation of the problem); (ii) numerical solution; and (iii) computer implementation of the method (rCADiff software). ${ }^{14}$ The solver based on the Runge-Kutta-Fehlberg method with adaptive step size control. ${ }^{15}$

\section{THE REACTIVE INTERDIFFUSION IN CU-SN SYSTEM, THE COMPARISON OF THE STEADY STATE AND EVOLUTIONARY SOLUTIONS}

Experimental, diffusion multiple method. The diffusion multiple technique is based on a multi-phase composite produced by appropriate bonding of different materials (alloys, IMCs, etc.). ${ }^{16,17}$ It allows investigating the diffusion processes between different phases, such as phase equilibria, kinetics of solid-state reactions, and mass transport. Moreover, the localized property measurements allow to map various material properties.
The diffusion multiples were produced as assemblies of selected pure metal blocks ( $\mathrm{Ag}, \mathrm{Cu}, \mathrm{Sn}$, and $\mathrm{Ni}$ ), while assuring that all materials were initially in intimate mechanical contact. ${ }^{18}$ For the fabrication of diffusion multiple samples, a titanium cylinder (20 mm diameter $\times 30 \mathrm{~mm}$ length) was cut by Electrical Discharge Machine. The resulting can was filled up with precut beams of $\mathrm{Ag}, \mathrm{Cu}, \mathrm{Ni}$, and $\mathrm{Sn}$. By using the hot isostatic pressing technique (HIP), the assembly was diffusion bonded. To achieve this, the Ti can was sealed and hot isostatic pressed for $1 \mathrm{~h}$ under the $T=210^{\circ} \mathrm{C}$ and $\mathrm{p}=180 \mathrm{MPa}$. A Paterson Gas-Medium High-Pressure High Temperature Testing System equipped with a control system was used for the HIP treatment of the Ti cylinder. Samples were obtained by cutting the cylinder into the coin shaped slices. In this way, multiple junctions of 3 and 2 different metals were obtained. Thermal treatment (annealing) of individual slices (samples) allowed studying of the IMC growth. The solid state reactions at $T=200^{\circ} \mathrm{C}$ were investigated in 

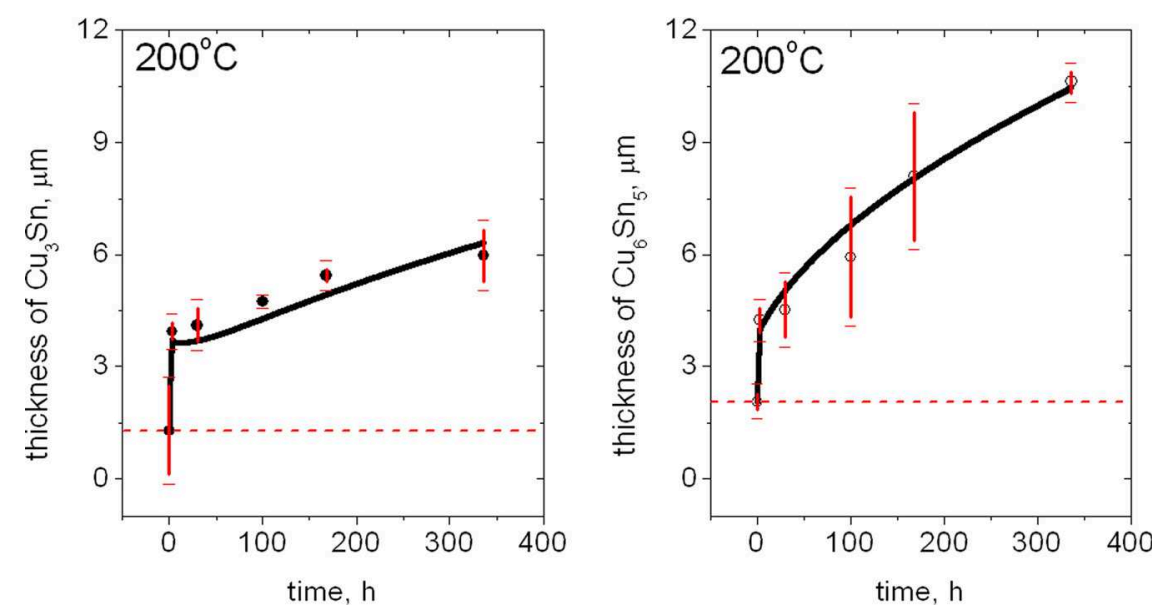

FIG. 7. (Color online) The growth of the intermetallic compounds $\mathrm{Cu}_{3} \mathrm{Sn}$ and $\mathrm{Cu}_{6} \mathrm{Sn}_{5}$ between pure $\mathrm{Cu}$ and $\mathrm{Sn}$ at $473 \mathrm{~K}$ in the diffusion multiple shown in Fig. 4. Comparison of the experimental results (points) with the rCADiff simulation (solid lines). The dashed line represents the initial thickness of IMC after HIP process (simulation not shown), the bars represent the three standard deviation from the mean experimental value. the time range from $3 \mathrm{~h}$ to 2 weeks $(336 \mathrm{~h})$. Figure 4 shows the overall view of the diffusion multiple after HIP process [Fig. 4(a)] and the cross sections of its $\mathrm{Cu}-\mathrm{Sn}$ zone after HIP process [Fig. 4(b)] and after annealing at $200{ }^{\circ} \mathrm{C}$ for $336 \mathrm{~h}$ [Fig. 4(c)].

The thermochemical data and mobilities used in this work are presented in Tables I and II, the interface thickness $\delta^{j}=5 \cdot 10^{-8}[\mathrm{~cm}]$.

Comparison of the quasi-stationary analytical and evolutionary solutions of the simultaneous growth of the two phases allows for a mathematical (formal) validation of the bivelocity method and its rCADiff numerical solution. Table II shows the mobilities and interdiffusion coefficients used in computations shown in Figs. 5-7. As required by the steady state analytical solution (Appendix D), the partial molar volumes were constant in whole system, $\Omega_{i}^{j}=10\left[\mathrm{~cm}^{3} \mathrm{~mol}^{-1}\right] \forall i, j$.

Figure 5 presents a comparison of the stationary solution and rCADiff simulation of the diffusion controlled, simultaneous growth of the intermetallic compounds $\mathrm{Cu}_{3} \mathrm{Sn}$ and $\mathrm{Cu}_{6} \mathrm{Sn}_{5}$ between pure $\mathrm{Cu}$ and $\mathrm{Sn}$ at $200^{\circ} \mathrm{C}$. It shows complete consistency of the analytical solution, set of analytical Eqs. (D15) and (D16), and the rCADiff method within the parabolic regime of the reaction, i.e., where simplified assumptions (required by the analytical approach) hold.

The validation of the bi-velocity method and its numerical solution is very effectively shown in the case when the thermodynamic equilibrium is reached (long reaction time). Because the parent metals $(\mathrm{Cu}$ and $\mathrm{Sn})$ are consumed with time, the system attains thermodynamic equilibrium at some point in time. This is a case, when the IMCs between the parent metals ( $\mathrm{Cu}$ and $\mathrm{Sn}$ ) have consumed all of the $\mathrm{Cu}$ (or $\mathrm{Sn}$ ). The evolution of the $\mathrm{Cu}-\mathrm{Sn}$ system till the thermodynamic equilibrium is presented in Fig. 6. It shows the evolution of the finite $\mathrm{Cu}-\mathrm{Sn}$ diffusion couple with an excess of $\mathrm{Sn}$. Three distinct reaction periods are visible: 1) simultaneous growth of IMCs between $\mathrm{Cu}$ and $\mathrm{Sn}(0<t<\sim 250 \mathrm{~h}$, until complete $\mathrm{Cu}$ consumption), 2) growth of $\mathrm{Cu}_{6} \mathrm{Sn}_{5}$ between $\mathrm{Cu}_{3} \mathrm{Sn}$ and $\mathrm{Sn}$ ( $\sim 250 \mathrm{~h}<t<\sim 500 \mathrm{~h}$, until complete $\mathrm{Cu}_{3} \mathrm{Sn}$ consumption), and 3 ) equilibrium of the system, where, given the initial composition of the couple and the temperature, at the point of equilibrium $\mathrm{Cu}_{6} \mathrm{Sn}_{5}$ and $\mathrm{Sn}$ are the only stable phases $(t>\sim 500 \mathrm{~h})$. These results demonstrate that bi-velocity method allows to simulate the entire reaction process over extended period of time involving growth and consumption of several phases.

Figure 7 shows the agreement between the experimental results and simulation of the simultaneous growth of the intermetallic compounds $\mathrm{Cu}_{3} \mathrm{Sn}$ and $\mathrm{Cu}_{6} \mathrm{Sn}_{5}$ in the diffusion multiple between pure $\mathrm{Cu}$ and $\mathrm{Sn}$. The process was divided into two time periods due to strong influence of the multiple fabrication stage ( $\mathrm{HIP}$ at $210^{\circ} \mathrm{C}$ ). During the initial period of the reaction (HIP period) the stress relaxation occurred and negative volume effect was observed. The initial HIP period of the reaction was simulated at $T=210^{\circ} \mathrm{C}$ assuming $D^{C u_{3} S n}=3.5 \cdot 10^{-10}$ and $D^{C u_{6} S n_{5}}=2.2 \cdot 10^{-10}$. During second period (diffusion annealing) the solid state reactions at $T=200^{\circ} \mathrm{C}$ were simulated. The values of interdiffusion coefficients used in computation are shown in Table II. ${ }^{19-21}$

\section{CONCLUSIONS}

In this paper, we presented the bi-velocity method and the effective use of the volume continuity equation:

1. We defined the volume-fixed frame of reference in the multicomponent and multiphase systems.

2. The volume continuity equation allows defining the material drift velocity, i.e., the lattice fixed coordinate axes for diffusion, Eq. (9).

3. The comparison of the steady state analytical and bivelocity (numerical implementation-rCADiff software) solutions during the parabolic regime of the reaction and when simplified assumptions (required by the analytical approach) hold shows the full consistency of presented methods.

4. The effective use of the method and practical applications in the binary, multiphase $\mathrm{Cu}-\mathrm{Sn}$ system were shown.

5. Presented results demonstrate that the bi-velocity method allows to simulate the whole reaction time (till the thermodynamic equilibrium is obtained).

6. We effectively computed the mass transport at nano-scale by considering the processes within the phase boundary zone $-\delta^{j}=0.5[\mathrm{~nm}], \forall j$.

7. Bi-velocity method allows quantitative description of the diffusion controlled solid-state reactions in the binary multiphase systems over the whole reaction period and 
from nano- to millimeter scale (the oxidation reactions, the diffusion coatings formation, etc.).

\section{ACKNOWLEDGMENTS}

This work has been supported by the Ministry of Higher Education and Science in Poland N N507 505138.

\section{APPENDIX A: THERMODYNAMIC FACTOR, COMPOSITION DEPENDENT INTERDIFFUSIVITY, AND INTEGRAL INTERDIFFUSION COEFFICIENT IN REACTING BINARY SYSTEM WITH LOCAL EQUILIBRIUM AT INTERFACES}

\section{Para-equilibrium at interfaces}

We do consider binary compound $\left(\alpha^{j}\right)$ shown in Figs. 1 and 3(b), within its homogeneity range:

1. Components mobilities and self-diffusivities obey the Nernst-Einstein relation: $D_{i}^{j}=B_{i}^{j} k T$.

2. Partial molar volumes are constant, $\Omega_{1}^{j}, \Omega_{2}^{j}=$ const., the overall molar volume of $j$-phase equals: $\Omega^{j}=\sum_{i} \Omega_{i}^{j} N_{i}^{j}$.

3. The pure components are the terminal phases $\left(\mu_{1}^{j-1}=g^{j-1}\right.$ and $\left.\mu_{2}^{j+1}=g^{j+1}\right)$ and para-equilibrium condition holds at both interfaces. ${ }^{22}$

4. The second derivative of the Gibbs free energy $\left[g^{\prime \prime j}=\partial \mu_{i}^{j} / \partial N_{i}^{j}=\partial^{2} g^{j} / \partial\left(N_{i}^{j}\right)^{2}\right]$ shows a linear dependence on the molar ratio.

We apply the Darken method ${ }^{6,23}$ to the problem of chemical interdiffusion in the growing $j$-layer. The molar flux relatively to the left boundary is the sum of diffusion and Darken fluxes,

$$
J_{2}^{j}={ }^{d} J_{2}^{j}+c_{2}^{j D} v^{j},
$$

where ${ }^{d} J_{i}^{j}=-B_{i}^{j} c_{i}^{j} \operatorname{grad} \mu_{i}^{j}$ and $\nu^{j}$ denote the diffusion flux due to the gradient of diffusion potential and drift flux in $j$ phase, respectively.

From the definition of components fluxes and using VCE, Eqs. (11) and (A1) one gets ${ }^{6,23}$

$$
\begin{aligned}
D_{v^{j}} & =-1 / \Omega_{c} \sum_{i} \Omega_{i}^{j d} J_{i}^{j} \\
& =B_{1}^{j} \Omega_{1}^{j} N_{1}^{j} \operatorname{grad} \mu_{1}^{j}+B_{2}^{j} \Omega^{2} N_{2}^{j} N_{2}^{j} \operatorname{grad} \mu_{2}^{j},
\end{aligned}
$$

where $N_{2}^{j}=c_{2}^{j} / c$ is the molar ratio and ${ }^{\Omega} N_{2}^{j}=\Omega_{i}^{j} / \Omega^{j}$ denotes the molar volume ratio.

Combining Eqs. (A1) and (A2),

$$
\begin{aligned}
J_{2}^{j} & =-B_{2}^{j} c_{2}^{j} \operatorname{grad} \mu_{2}^{j}+c_{2}^{j}\left(B_{1}^{j} \Omega_{1}^{j} N_{1}^{j} \operatorname{grad} \mu_{1}^{j}+B_{2}^{j} \Omega_{2} N_{2}^{j} N_{2}^{j} \operatorname{grad} \mu_{2}^{j}\right) \\
& =-c_{2}^{j}\left(\left(1-{ }^{\Omega} N_{2}^{j} N_{2}^{j}\right) B_{2}^{j} \operatorname{grad} \mu_{2}^{j}-B_{1}^{j}{ }^{2} N_{1}^{j} N_{1}^{j} \operatorname{grad} \mu_{1}^{j}\right) .
\end{aligned}
$$

The Gibbs-Dühem $\left(N_{1}^{j} \mathrm{~d} \mu_{1}^{j}=-N_{2}^{j} \mathrm{~d} \mu_{2}^{j}\right)$ and Euler $\left({ }^{\Omega} N_{1}^{j} N_{1}^{j}+{ }^{\Omega} N_{2}^{j} N_{2}^{j}=\Omega_{1}^{j} c_{1}^{j}+\Omega_{2}^{j} c_{2}^{j}=1\right)$ relations introduced into Eq. (A3) result in

$$
\begin{aligned}
J_{2}^{j} & =-\left(B_{2}^{j} N_{1}^{j}+B_{1}^{j} N_{2}^{j}\right) \Omega^{\Omega} N_{1}^{j} c_{2}^{j} \operatorname{grad} \mu_{2}^{j} \\
& =-\left(B_{2}^{j} N_{1}^{j}+B_{1}^{j} N_{2}^{j}\right)\left(\frac{\partial \mu_{2}^{j}}{\partial N_{2}^{j}}\right){ }^{2} N_{1}^{j} c_{2}^{j} \operatorname{grad} N_{2}^{j} .
\end{aligned}
$$

\section{Approximations}

When the partial molar volumes are equal, the overall molar concentration is non-variant ( $c=$ const) and Eq. (A4) takes Fick'ian form,

$$
J_{2}^{j}=-\left(B_{2}^{j} N_{1}^{j}+B_{1}^{j} N_{2}^{j}\right) N_{2}^{j}\left(\frac{\partial \mu_{2}^{j}}{\partial N_{2}^{j}}\right) \operatorname{grad} c_{2}^{j}=-\tilde{D}^{j} \operatorname{grad} c_{2}^{j} .
$$

When thermodynamical data are available the (chemical) interdiffusion coefficient follows from above relation. In most cases, it is not so and one must estimate the thermodynamical term in Eq. (A5). The essence is the proper evaluation of the second derivative of the Gibbs potential (free energy) in each phase.

\section{Approximation of the composition dependence of the chemical potential}

In the binary system at constant temperature and when stresses are negligible, the integral and differential forms of the Gibbs relations become

$$
\begin{gathered}
g^{j}=N_{1}^{j} \mu_{1}^{j}+N_{2}^{j} \mu_{2}^{j}, \\
\mathrm{~d} g^{j}=\mu_{1}^{j} \mathrm{~d} N_{1}^{j}+\mu_{2}^{j} \mathrm{~d} N_{2}^{j}=\left(\mu_{2}^{j}-\mu_{1}^{j}\right) \mathrm{d} N_{2}^{j},
\end{gathered}
$$

where $g^{j}$ is the Gibbs free energy, $\mu_{1}^{j}$ and $\mu_{2}^{j}$ denote the chemical potentials, and we used identity $\mathrm{d} N_{1}^{j}=-\mathrm{d} N_{2}^{j}$. The Gibbs and the chemical (diffusion) potentials are expressed per one atom, for example, $g^{j}=G^{j} / N_{A}$. It will be convenient here to use the following form of the differential Gibbs relation, Eq. (A7):

$$
g^{\prime j}=\frac{\partial g^{j}}{\partial N_{2}^{j}}=\mu_{2}^{j}-\mu_{1}^{j} .
$$

The all quantities in Eqs. (A6)-(A8) depend on time and position.

Equation (A8) allows to calculate the dependence of the chemical potential on the molar ratio,

$$
\frac{\partial \mu_{2}^{j}}{\partial N_{2}^{j}}=\frac{\partial^{2} g^{j}}{\partial\left(N_{2}^{j}\right)^{2}}+\frac{\partial \mu_{1}^{j}}{\partial N_{2}^{j}}=g^{\prime \prime j}+\frac{\partial \mu_{1}^{j}}{\partial N_{2}^{j}}
$$

From the Gibbs Duheme relation $\left(N_{1}^{j} \mathrm{~d} \mu_{1}^{j}=-N_{2}^{j} \mathrm{~d} \mu_{2}^{j}\right)$ it follows also

$$
\frac{\partial \mu_{1}^{j}}{\partial N_{2}^{j}}=-\frac{N_{2}^{j}}{N_{1}^{j}} \frac{\partial \mu_{2}^{j}}{\partial N_{2}^{j}}
$$

Combining Eqs. (A9) and (A10) the composition dependence of the chemical potential is given by 


$$
\left(1+\frac{N_{2}^{j}}{N_{1}^{j}}\right) \frac{\partial \mu_{2}^{j}}{\partial N_{2}^{j}}=\frac{1}{N_{1}^{j}} \frac{\partial \mu_{2}^{j}}{\partial N_{2}^{j}}=g^{\prime \prime j} .
$$

From Eq. (A11) the composition dependence of the chemical potentials equals,

$$
\frac{\partial \mu_{2}^{j}}{\partial N_{2}^{j}}=N_{1}^{j} g^{\prime \prime j} \quad \text { and } \quad \frac{\partial \mu_{1}^{j}}{\partial N_{1}^{j}}=N_{2}^{j} g^{\prime \prime j} .
$$

Upon substituting Eq. (A12) into Eq. (A5) we have

$$
\tilde{D}^{j}=\left(D_{2}^{j} N_{1}^{j}+D_{1}^{j} N_{2}^{j}\right) \frac{N_{1}^{j} N_{2}^{j}}{k T} g^{\prime \prime j}=\left(B_{2}^{j} N_{1}^{j}+B_{1}^{j} N_{2}^{j}\right) N_{1}^{j} N_{2}^{j} g^{\prime \prime j} .
$$

Because we use the linear approximation within the $j$-phase homogeneity range, the second derivative of the Gibbs potential equals

$$
g^{\prime \prime j}=\frac{\partial^{2} g^{j}}{\partial\left(N_{2}^{j}\right)^{2}} \cong \frac{\frac{\partial g^{j}}{\partial N_{2}^{j}}(R)-\frac{\partial g^{j}}{\partial N_{2}^{j}}(L)}{{ }^{e q} N_{2}^{j}(R)-{ }^{e q} N_{2}^{j}(L)}=\text { const. }
$$

In the following, we will assess Eq. (A14) for the different boundary conditions.

\section{Para-equilibrium at terminal interfaces of $\mathbf{j}$-phase}

The first derivatives of the Gibbs free energy, $g^{j}$, can be estimated by introducing the para-equilibrium conditions at both interfaces shown in Fig. 1. Namely, by noting that A is not mobile (and solved) in B as well as in AlB interface and vice versa. Using now the integral form of Gibbs relation, Eqs. (A6) and (A8) we get

$$
\begin{aligned}
& \frac{\partial g^{j}}{\partial N_{2}}(R)={ }^{e q} \mu_{2}^{j}(R)-{ }^{e q} \mu_{1}^{j}(R) \stackrel{\mu_{2}^{j}(R)=g^{j+1}}{=} g^{j+1}-\frac{g^{j}-{ }^{e q} N_{2}^{j}(R) g^{j+1}}{{ }^{e q} N_{1}^{j}(R)}=-\frac{g^{j}-g^{j+1}}{e q N_{1}^{j}(R)}, \\
& \frac{\partial g^{j}}{\partial N_{2}}(L)={ }^{e q} \mu_{2}^{j}(L)-{ }^{e q} \mu_{1}^{j}(L) \stackrel{g^{j-1}=\mu_{1}^{j}(L)}{=} \frac{g^{j}-{ }^{e q} N_{1}^{j}(L) g^{j-1}}{{ }^{e q} N_{2}^{j}(L)}-g^{j-1}=\frac{g^{j}-g^{j-1}}{{ }^{e q} N_{2}^{j}(L)} .
\end{aligned}
$$

By combining Eqs. (A14) and (A15) we get

$$
\begin{aligned}
g^{\prime \prime j} & =-\left[\frac{g^{j}-g^{j+1}}{e q N_{1}^{j}(R)}-\frac{g^{j}-g^{j-1}}{e q N_{2}^{j}(L)}\right] / \Delta^{e q} N_{2}^{j} \\
& =-\frac{\Delta g^{j}}{{ }^{e q} N_{1}^{j}(R)^{e q} N_{2}^{j}(L) \Delta^{e q} N_{2}^{j}}=\text { const. },
\end{aligned}
$$

where $\Delta g^{j}=\left(g^{j}-g^{j+1}\right)^{e q} N_{2}^{j}(L)+\left(g^{j}-g^{j-1}\right)^{e q} N_{1}^{j}(R)$.

From Eqs. (A15) and (A16), the interdiffusion coefficient in the binary reacting system under the paraequilibrium conditions at both interfaces equals

$$
\begin{aligned}
\tilde{D}^{j} & =-\left(D_{2}^{j} N_{1}^{j}+D_{1}^{j} N_{2}^{j}\right) \frac{N_{1}^{j} N_{2}^{j}}{e q N_{1}^{j}(R)^{e q} N_{2}^{j}(L)} \frac{\Delta g^{j}}{k T \Delta^{e q} N_{2}^{j}} \\
& =-\left(B_{2}^{j} N_{1}^{j}+B_{1}^{j} N_{2}^{j}\right) \frac{N_{1}^{j} N_{2}^{j}}{{ }^{e q} N_{1}^{j}(R)^{e q} N_{2}^{j}(L)} \frac{\Delta g^{j}}{\Delta^{e q} N_{2}^{j}} .
\end{aligned}
$$

When we consider compound showing low nonstoichiometry $N_{1}^{j}(x) \cong N_{1}^{j}(R)$ and $N_{2}^{j}(x) \cong N_{2}^{j}(L)$, it becomes

$$
\tilde{D}^{j}=-\left(B_{2}^{j} N_{1}^{j}+B_{1}^{j} N_{2}^{j}\right) \frac{\Delta g^{j}}{\Delta N_{2}^{j}},
$$

and integral interdiffusivity is given by

$$
\tilde{D}_{\text {Int }}^{j}=-\left(B_{2}^{j} N_{1}^{j}+B_{1}^{j} N_{2}^{j}\right) \Delta g^{j} .
$$

\section{Orthoequilibrium at interfaces}

Figure 2: when both components are mobile, we assume the ortho-equilibrium at the interfaces between reacting IMC's $\left(\alpha^{j} \mid \alpha^{j+1}\right)$. For example, at the right interface of the $j$ phase the orthoequilibrium implies

$$
{ }^{e q} \mu_{2}^{j}(R)={ }^{e q} \mu_{2}^{j+1}(L) \quad \text { and } \quad{ }^{e q} \mu_{1}^{j}(R)={ }^{e q} \mu_{1}^{j+1}(L) .
$$

Using the integral form of the Gibbs relation on the both sides of each interface and introducing the LE conditions, e.g., Eq. (A20), we have the four relations and four unknown chemical potentials,

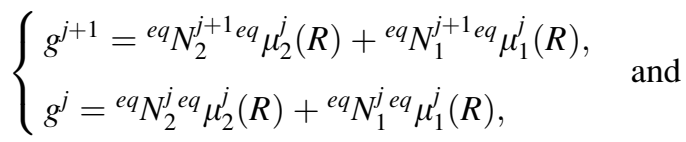

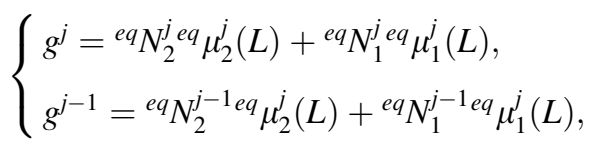

where the all molar ratios and chemical potentials are equilibrium values at respective phase boundaries.

Using relations (A21) we can compute the " $\mu_{2}^{j}-\mu_{1}^{j}$ ", term in Eq. (A8). By substracting relations in Eq. (A21), eliminating the molar ratio of the first component $\left(N_{1}^{j}=1-N_{2}^{j}\right)$ and after laborious transformations we get

$$
\begin{aligned}
& g^{\prime j}(R)={ }^{e q} \mu_{2}^{j}(R)-{ }^{e q} \mu_{1}^{j}(R)=-\frac{g^{j+1}-g^{j}}{{ }^{e q} N_{2}^{j}(R)-{ }^{e q} N_{2}^{j+1}(L)} \\
& g^{\prime j}(L)={ }^{e q} \mu_{2}^{j}(L)-{ }^{e q} \mu_{1}^{j}(L)=-\frac{g^{j}-g^{j-1}}{{ }^{e q} N_{2}^{j-1}(R)-{ }^{e q} N_{2}^{j}(L)} .
\end{aligned}
$$

By combining Eqs. (A14) and (A22), the second derivative of the Gibbs potential equals 
$g^{\prime \prime j}=-\frac{1}{\Delta^{e q} N_{2}^{j}}\left[\frac{g^{j+1}-g^{j}}{e q N_{2}^{j}(R)-{ }^{e q} N_{2}^{j+1}(L)}+\frac{-g^{j}+g^{j-1}}{e q N_{2}^{j-1}(R)-{ }^{e q} N_{2}^{j}(L)}\right]$,

and in the binary reacting system with orthoequilibrium at both interfaces (Fig. 2) the composition dependent interdiffusion coefficient equals

$$
\begin{aligned}
\tilde{D}^{j}= & -\left(B_{2}^{j} N_{1}^{j}+B_{1}^{j} N_{2}^{j}\right) \frac{N_{1}^{j} N_{2}^{j}}{\Delta^{e q} N_{2}^{j}} \\
& \left(\frac{g^{j+1}-g^{j}}{e q N_{2}^{j}(R)-e q N_{2}^{j+1}(L)}+\frac{-g^{j}+g^{j-1}}{e q N_{2}^{j-1}(R)-e q N_{2}^{j}(L)}\right) .
\end{aligned}
$$

\section{APPENDIX B: NON-EQUILIBRIUM CONDITIONS AT INTERFACES (ORTHO-EQUILIBRIUM AT BOTH INTERFACES OF PHASE BOUNDARY ZONE)}

The transport problems in materials science are frequently solved assuming the "trouble-free" conditions at phase boundaries, for example, orthoequilibrium as in Eq. (A20). Consequently, conditions at phase boundaries are often "non-physical:"

1. the thickness and transport properties of the phase boundary zone are not considered;

2. it is assumed that rate of mass transport through all $\alpha^{j} \mid \alpha^{j+1}$ phase boundaries is much faster than in the bulk and, consequently;

3. the transport through interface is considered as a process without the driving force.

To envisage the formula for the flux through the phase boundary we introduce the concept of the phase boundary zone, Fig. 3(c). One can now consider the deviation from LE in phase boundary zone and evaluate the "new," shifted nonequilibrium values of chemical potentials at interfaces of this zone adjacent to neighboring phases. To do so we assume

1. Orthoequilibrium [continuity of the chemical potential, Fig. 3(c)]:

$$
\mu_{i}^{j}(R)=\mu_{i}^{j}(I, L) \quad \text { and } \quad \mu_{i}^{j+1}(L)=\mu_{i}^{j}(I, R) .
$$

2. Only the small deviations of the chemical potentials (linear approximation).

3. The non-variant: thickness, the mobilities of components, and their concentrations in every phase boundary zone,

$$
\begin{aligned}
B_{2}^{j}(I) & =\text { const, } c_{i}^{j}(I)=\left\langle c_{i}^{j}(I)\right\rangle=\text { constand } \\
\delta^{j} & =\text { const. }
\end{aligned}
$$

4. The Nernst-Planck diffusion flux in the phase boundary zone

$$
\begin{aligned}
J_{i}^{j}(I) & ={ }^{d} J_{i}^{j}(I)=-B_{i}^{j}(I) c_{i}^{j}(I) \operatorname{grad} \mu_{i}^{j}(I) \\
& =B_{i}^{j}(I) c_{i}^{j}(I) \frac{\mu_{i}^{j}(R)-\mu_{i}^{j+1}(L)}{\delta^{j}} .
\end{aligned}
$$

The driving force in Eq. (68): $\quad F_{i}^{j}(I)=\left\{\mu_{i}^{j}(t, R)\right.$ $\left.-\mu_{i}^{j+1}(t, L)\right\} / \delta^{j}$ is due to the transport processes through the phase boundary and depends on thermodynamics of neighboring phases. The chemical potentials are the non-equilibrium values at the interfaces of neighboring phases. From Eq. (55) and Gibbs-Duheme relation, one can compute the time dependent deviations of the chemical potentials at interfaces

$$
\begin{aligned}
& \mathrm{d} \mu_{1}^{j}(R)=\left({ }^{e q} N_{1}^{j}-N_{1}^{j}(R)\right) N_{2}^{j}(R) g^{\prime \prime j} \quad \text { and } \\
& \mathrm{d} \mu_{2}^{j}(R)=\left({ }^{e q} N_{2}^{j}-N_{2}^{j}(R)\right) N_{1}^{j}(R) g^{\prime \prime j} .
\end{aligned}
$$

The time dependent chemical potentials of the first component at interfaces of the $j$-th phase boundary zone equal

$$
\begin{aligned}
& \mu_{1}^{j}(R)=\mu_{1}^{j}(I, L)={ }^{e q} \mu_{1}^{j}(R)+\mathrm{d} \mu_{1}^{j}(R), \\
& \mu_{1}^{j+1}(L)=\mu_{1}^{j}(I, R)={ }^{e q} \mu_{1}^{j+1}(L)+\mathrm{d} \mu_{1}^{j+1}(L) .
\end{aligned}
$$

From Eqs. (B1) and (B3) and, because the equilibrium values of the chemical potential in neighboring phases equal $\left.{ }^{e q} \mu_{i}^{j}(R)={ }^{e q} \mu_{i}^{j+1}(L)\right)$, the approximated driving force in the $j$-th boundary zone is given by

$$
F_{1}^{j}(t, I)=-\operatorname{grad} \mu_{1}^{j}(t, I) \cong-\frac{\left({ }^{e q} N_{1}^{j+1}-N_{1}^{j+1}(L)\right) N_{2}^{j+1}(L) g^{\prime \prime j+1}-\left({ }^{e q} N_{1}^{j}-N_{1}^{j}(R)\right) N_{2}^{j}(R) g^{\prime \prime j}}{\delta^{j}} .
$$

The diffusion flux in the phase boundary zone is now determined by the deviations from equilibrium at interfaces and by the transport properties of the phase boundary zone,

$$
\begin{aligned}
& J_{1}^{j}(I) \cong-\frac{B_{1}^{j}(I) c_{1}^{j}(I)}{\delta^{j}}\left(\left({ }^{e q} N_{1}^{j+1}-N_{1}^{j+1}(L)\right) N_{2}^{j+1}(L) g^{\prime \prime j+1}-\left({ }^{e q} N_{1}^{j}-N_{1}^{j}(R)\right) N_{2}^{j}(R) g^{\prime \prime j}\right), \\
& J_{2}^{j}(I) \cong-\frac{B_{2}^{j}(I) c_{2}^{j}(I)}{\delta^{j}}\left(\left({ }^{e q} N_{1}^{j+1}-N_{1}^{j+1}(L)\right) N_{1}^{j+1}(L) g^{\prime \prime j+1}-\left({ }^{e q} N_{2}^{j}-N_{2}^{j}(R)\right) N_{1}^{j}(R) g^{\prime \prime j}\right) .
\end{aligned}
$$




\section{APPENDIX C: STEADY-STATE GROWTH}

In order to obtain the linear formula for the flux through the phase boundary zone, we consider the deviation from LE in phase boundary zone and evaluate the "new" values of chemical potentials at interfaces of the phase boundary zone adjacent to neighboring phases. To do so we assume:

1. At those two abstract interfaces (borders of the phase boundary), we postulate LE assumption (continuity of the chemical potential),

$$
\mu_{i}^{j}(R)=\mu_{i}^{j}(I, \text { left side }) \text { and } \mu_{i}^{j+1}(L)=\mu_{i}^{j}(\text { I, right side }) .
$$

2. We limit ourself to analyze the small deviations of the chemical potentials (linear approximation).

3. At isothermal-isobaric conditions the Gibbs-Düchem relation holds in the phase boundary zone,

$$
N_{1}(I) \mathrm{d} \mu_{1}^{j}(I)+N_{2}(I) \mathrm{d} \mu_{2}^{j}(I)=0 .
$$

4. The integral form of Gibbs relation (e.g., an energy per atom) in the phase boundary zone,

$$
N_{1}^{j} \mu_{1}^{j}+N_{2}^{j} \mu_{2}^{j}=g^{j} \text { and } \frac{\partial g^{j}}{\partial N_{2}^{j}} \equiv g^{\prime j}=\mu_{2}^{j}-\mu_{1}^{j} .
$$

5. The non-variant: thickness, mobilities, and average concentrations of components in every phase boundary zone,

$$
B_{2}^{j}(I)=\text { const. },\left\langle c_{i}^{j}(I)\right\rangle=\text { const. } \quad \text { and } \quad \delta^{j}=\text { const } .
$$

6. Consequently, the Nernst-Planck flux becomes

$$
\begin{aligned}
J_{i}^{j}(I) & ={ }^{d} J_{i}^{j}(I)=-B_{i}^{j}(I)\left\langle c_{i}^{j}(I)\right\rangle \operatorname{grad} \mu_{i}^{j}(I) \\
& \cong B_{i}^{j}(I)\left\langle c_{i}^{j}(I)\right\rangle \frac{\mu_{i}^{j}(R)-\mu_{i}^{j+1}(L)}{\delta^{j}}
\end{aligned}
$$

The key point now is to compute the driving forces in Eq. (B1), $\quad F_{i}^{j}=\left(\mu_{i}^{j}(t, R)-\mu_{i}^{j+1}(t, L)\right) / \delta^{j}$. The chemical potentials are the non-equilibrium values at interfaces of neighboring phases. From Eq. (C1) and Gibbs-Duheme relation, one can compute the local, time dependent deviation from equilibrium of the chemical potentials at interfaces

$$
\mathrm{d} \mu_{1}^{j}(t)=-N_{2}^{j} \mathrm{~d} g^{\prime j}(t) \quad \text { and } \quad \mathrm{d} \mu_{2}^{j}(t)=N_{1}^{j} \mathrm{~d} g^{\prime j}(t) .
$$

The time dependent chemical potentials of the first component at interfaces of the $j$-th phase boundary zone equal

$$
\begin{aligned}
\mu_{1}^{j}(t, R) & =\mu_{1}^{j}(t, I \text {-left })=\mu_{1}^{j}(I \text {-left-LE })+\mathrm{d} \mu_{1}^{j}(t) \\
= & \mu_{1}^{j}(I \text {-left-LE })-N_{2}^{j} \mathrm{~d} g^{\prime j}(t), \\
\mu_{1}^{j+1}(t, L) & =\mu_{1}^{j}(t, I \text {-right })=\mu_{1}^{j}(I \text {-right-LE })+\mathrm{d} \mu_{1}^{j+1}(t) \\
& =\mu_{1}^{j}(I \text {-right-LE })-N_{2}^{j+1} \mathrm{~d} g^{\prime j+1}(t),
\end{aligned}
$$

where at equilibrium: $\mu_{i}^{j}(I$-left-LE $)=\mu_{i}^{j}(I$-right-LE $)$ $=\mu_{i}^{j}(R$-LE $)=\mu_{i}^{j+1}(L-\mathrm{LE})$. Consequently, from Eqs. (B1)-(B3), the approximated driving force in the $j$-th boundary zone equals

$$
F_{1}^{j}(t, I)=-\operatorname{grad} \mu_{1}^{j}(t, I) \cong \frac{N_{2}^{j+1} \mathrm{~d} g^{\prime j+1}-N_{2}^{j} \mathrm{~d} g^{\prime j}}{\delta^{j}},
$$

and the diffusion flux in the phase boundary zone is now determined by the deviations from equilibrium at interfaces and physico-chemical properties of the phase boundary zone,

$$
J_{1}^{j}(I) \cong B_{1}^{j}(I)\left\langle c_{1}^{j}(I)\right\rangle \frac{N_{2}^{j+1} \mathrm{~d} g^{\prime j+1}-N_{2}^{j} \mathrm{~d} g^{\prime j}}{\delta^{j}} .
$$

The chemical potentials of the second component at $j$-th phase boundary interfaces equal

$$
\begin{aligned}
\mu_{2}^{j}(t, R) & =\mu_{2}^{j}(t, I \text {-left })=\mu_{2}^{j}(I \text {-left-LE })+\mathrm{d} \mu_{2}^{j}(t) \\
& =\mu_{2}^{j}(I \text {-left-LE })+N_{1}^{j} \mathrm{~d} g^{\prime j}(t), \\
\mu_{2}^{j+1}(t, L) & =\mu_{2}^{j}(t, I \text {-right })=\mu_{2}^{j}(I \text {-right-LE })+\mathrm{d} \mu_{2}^{j+1}(t) \\
& =\mu_{2}^{j}(I \text {-right-LE })+N_{1}^{j+1} \mathrm{~d} g^{\prime j+1}(t) .
\end{aligned}
$$

The driving force and diffusion flux of the second component in the $j$-th boundary zone equal

$$
\begin{aligned}
& F_{2}^{j}(t, I)=-\operatorname{grad} \mu_{2}^{j}(t, I) \cong \frac{N_{1}^{j} \mathrm{~d} g^{\prime j}-N_{1}^{j+1} \mathrm{~d} g^{\prime j+1}}{\delta^{j}}, \\
& J_{2}^{j}(t, I) \cong B_{2}^{j}(I)\left\langle c_{2}^{j}(I)\right\rangle \frac{N_{1}^{j} \mathrm{~d} g^{\prime j}-N_{1}^{j+1} \mathrm{~d} g^{\prime j+1}}{\delta^{j}} .
\end{aligned}
$$

\section{APPENDIX D: ANALYTICAL SOLUTION (EXAMPLE)}

We reduced the problem by assuming (a) the equal partial molar volumes of all species in a given phase: $\Omega_{1}^{j}=\Omega_{2}^{j}=\Omega^{j}=$ const.; (b) the linear deviations of chemical potentials from their LE values (by substituting those deviations of chemical potential by the deviations of concentrations). Below we shall consider binary system and the formation of two intermetallic compounds between the single component terminal phases: $i=1,2$ and $j=0,1,2$ in Fig. 1. Additionally, we assume the equal molar volumes of each phase: $\Omega=\Omega^{0}=\Omega^{1}=\Omega^{2}=\Omega^{3}$. Thus, the equation of volume velocity, Eq. (20) reduces to

$$
v^{j}=\dot{X}^{j}+\Omega \sum_{i} J_{i}^{j}(I) .
$$

If the left terminal phase is fixed at its right boundary (the interphase velocity equals zero: $X^{0}(t)=0$ ), the volume velocities in the phases forming the mixture are equal [follows from Eq. (10)].

The deviation of chemical potential at interfaces can be expressed by the deviation of concentration using Eq. (C3) second derivative of Gibbs potential: $\partial g^{\prime j} / \partial N_{2}^{j}=g^{\prime \prime}$. From this definition,

$$
\mathrm{d} g^{\prime j}(t)=g^{\prime \prime j} \mathrm{~d} N_{2}^{j}(t)
$$

The fluxes at interfaces of $\alpha^{j}$ and $\alpha^{j+1}$, Eqs. (33) and (34), are now expressed by 


$$
\begin{aligned}
& { }^{d} J_{2}^{1}(R)=h_{R}^{1} g^{\prime \prime 1} \mathrm{~d} N_{2}^{1}(t, R)-f_{R}^{1} g^{\prime \prime 2} \mathrm{~d} N_{2}^{2}(t, L), \\
& { }^{d} J_{2}^{1}(L)=h_{L}^{1} g^{\prime \prime 0} \mathrm{~d} N_{2}^{0}(t, R)-f_{L}^{1} g^{\prime \prime 1} \mathrm{~d} N_{2}^{1}(t, L), \\
& { }^{d} J_{2}^{2}(R)=h_{R}^{2} g^{\prime \prime 2} \mathrm{~d} N_{2}^{2}(t, R)-f_{R}^{2} g^{\prime \prime \prime} \mathrm{d} N_{2}^{3}(t, L), \\
& { }^{d} J_{2}^{2}(L)=h_{L}^{2} g^{\prime \prime 1} \mathrm{~d} N_{2}^{1}(t, R)-f_{L}^{2} g^{\prime \prime 2} \mathrm{~d} N_{2}^{2}(t, L),
\end{aligned}
$$

where

$$
\begin{aligned}
f_{R}^{1} & =\frac{N_{1}^{1} N_{1}^{2} B_{2}^{1}(I)\left\langle c_{2}^{1}(I)\right\rangle+N_{2}^{1} N_{2}^{2} B_{1}^{1}(I)\left\langle c_{1}^{1}(I)\right\rangle}{\delta^{1}} ; \\
h_{R}^{1} & =\frac{N_{1}^{1} N_{1}^{1} B_{2}^{1}(I)\left\langle c_{2}^{1}(I)\right\rangle+N_{2}^{1} N_{2}^{1} B_{1}^{1}(I)\left\langle c_{1}^{1}(I)\right\rangle}{\delta^{1}} \\
f_{L}^{1} & =\frac{N_{1}^{1} N_{1}^{1} B_{2}^{0}(I)\left\langle c_{2}^{0}(I)\right\rangle+N_{2}^{1} N_{2}^{1} B_{1}^{0}(I)\left\langle c_{1}^{0}(I)\right\rangle}{\delta^{0}} ; \\
h_{L}^{1} & =\frac{N_{1}^{0} N_{1}^{1} B_{2}^{0}(I)\left\langle c_{2}^{0}(I)\right\rangle+N_{2}^{0} N_{2}^{1} B_{1}^{0}(I)\left\langle c_{1}^{0}(I)\right\rangle}{\delta^{0}} \\
f_{R}^{2} & =\frac{N_{1}^{2} N_{1}^{3} B_{2}^{2}(I)\left\langle c_{2}^{2}(I)\right\rangle+N_{2}^{2} N_{2}^{3} B_{1}^{2}(I)\left\langle c_{1}^{2}(I)\right\rangle}{\delta^{2}} ; \\
h_{R}^{2} & =\frac{N_{1}^{2} N_{1}^{2} B_{2}^{2}(I)\left\langle c_{2}^{2}(I)\right\rangle+N_{2}^{2} N_{2}^{2} B_{1}^{2}(I)\left\langle c_{1}^{2}(I)\right\rangle}{\delta^{2}} \\
f_{L}^{2} & =\frac{N_{1}^{2} N_{1}^{2} B_{2}^{1}(I)\left\langle c_{2}^{1}(I)\right\rangle+N_{2}^{2} N_{2}^{2} B_{1}^{1}(I)\left\langle c_{1}^{1}(I)\right\rangle}{\delta^{1}} ; \\
h_{L}^{2} & =\frac{N_{1}^{1} N_{1}^{2} B_{2}^{1}(I)\left\langle c_{2}^{1}(I)\right\rangle+N_{2}^{1} N_{2}^{2} B_{1}^{1}(I)\left\langle c_{1}^{1}(I)\right\rangle}{\delta^{1}} .
\end{aligned}
$$

In such system, three interfaces exists (para-equilibrium at the $\alpha_{0} \mid \alpha_{1}$ and $\alpha_{3} \mid \alpha_{4}$ and local equilibrium at $\alpha_{1} \mid \alpha_{2}$ phase boundaries) and four unknowns (composition deviations) must be found at the $\alpha_{0}\left|\alpha_{1}, \alpha_{1}\right| \alpha_{2}$, and $\alpha_{3} \mid \alpha_{4}$ phase boundaries. We want to compute the thickness of the growing phases, thus, we must relate fluxes with the thickness of $\alpha_{1}$ and $\alpha_{2}$. From Eq. (3),

$$
{ }^{V} J_{2}^{j}=-\tilde{D}_{2}^{j} \Delta N_{2}^{j} / X^{j}(t)
$$

where $X^{j}(t)$ denotes the thickness (not the position) of the $j$-phase and the molar flux is given by

$$
{ }^{d} J_{2}^{j}=-\frac{\tilde{D}_{2}^{j} \Delta N_{2}^{j}(t)}{\Omega_{2}^{j} X^{j}(t)} \stackrel{\Omega_{i}^{j}=\Omega^{j}}{=}-\frac{\tilde{D}_{2}^{j} c^{j}}{X^{j}(t)}\left(N_{2}^{j}(t, R)-N_{2}^{j}(t, L)\right) .
$$

Taking into account that we consider the deviations of molar ratios at interfaces, we have

$$
\begin{aligned}
\Delta N_{2}^{j}(t) & =N_{2}^{j}(t, R)-N_{2}^{j}(t, L) \\
& =N_{2}^{j}(R, L E)+\mathrm{d} N_{2}^{j}(t, R)-N_{2}^{j}(L, L E)-\mathrm{d} N_{2}^{j}(t, L) \\
& =\Delta N_{2}^{j}(L E)+\mathrm{d} N_{2}^{j}(t, R)-\mathrm{d} N_{2}^{j}(t, L) \\
& =\Delta^{e q} N_{2}^{j}+\mathrm{d} N_{2}^{j}(t, R)-\mathrm{d} N_{2}^{j}(t, L) .
\end{aligned}
$$

Combining Eqs. (D10) and (D11), we expressed the flux in the growing phase by the local deviations of composition from their LE values

$$
{ }^{d} J_{2}^{j}=-\frac{\tilde{D}^{j} c^{j} \Delta^{e q} N_{2}^{j}}{X^{j}(t)}-\frac{\tilde{D}^{j} c^{j}}{X^{j}(t)}\left[\mathrm{d} N_{2}^{j}(t, R)-\mathrm{d} N_{2}^{j}(t, L)\right] .
$$

In the concise form we have

$$
{ }^{d} J_{2}^{1}=-\frac{\tilde{D}^{1} \Delta^{e q} c_{2}^{1}}{X^{j}(t)}-\frac{\tilde{D}^{1} c^{1}}{X^{j}(t)}\left(\mathrm{d} N_{2}^{1}(R)-\mathrm{d} N_{2}^{1}(L)\right)
$$

$$
{ }^{d} J_{2}^{2}=-\frac{\tilde{D}^{2} \Delta^{e q} c_{2}^{2}}{X^{2}(t)}-\frac{\tilde{D}^{2} c^{2}}{X^{2}(t)}\left(\mathrm{d} N_{2}^{2}(R)-\mathrm{d} N_{2}^{2}(L)\right) .
$$

The linear system of 6 equations, Eqs. (D3)-(D7), (D13), and (D14) can be reduced. Because we consider steady state process and position independent fluxes: $J_{2}^{j}=J_{2}^{j}(t, L)=J_{2}^{j}(t, R)$, we finally get

$$
\begin{gathered}
-\frac{\tilde{D}^{1} \Delta^{e q} c_{2}^{1}}{X^{j}(t)}-\frac{\tilde{D}^{1} c^{1}}{X^{j}(t)}\left(\mathrm{d} N_{2}^{1}(R)-\mathrm{d} N_{2}^{1}(L)\right) \\
=-f_{L}^{1} g^{\prime \prime 1} \mathrm{~d} N_{2}^{1}(t, L)-f_{L}^{1} g^{\prime \prime 1} \mathrm{~d} N_{2}^{1}(t, L) \\
=h_{R}^{1} g^{\prime \prime 1} \mathrm{~d} N_{2}^{1}(t, R)-f_{R}^{1} g^{\prime \prime 2} \mathrm{~d} N_{2}^{2}(t, L),
\end{gathered}
$$

$$
h_{L}^{2} g^{\prime \prime 1} \mathrm{~d} N_{2}^{1}(t, R)-f_{L}^{2} g^{\prime \prime 2} \mathrm{~d} N_{2}^{2}(t, L)=h_{R}^{2} g^{\prime \prime 2} \mathrm{~d} N_{2}^{2}(t, R),
$$$$
h_{R}^{2} g^{\prime \prime 2} \mathrm{~d} N_{2}^{2}(t, R)=-\frac{\widetilde{D^{2}} \Delta^{e q} c_{2}^{2}}{X^{2}(t)}-\frac{\widetilde{D^{2} c^{2}}}{X^{2}(t)}\left(\mathrm{d} N_{2}^{2}(R)-\mathrm{d} N_{2}^{2}(L)\right),
$$

where the thickness of the sub-layers is computed from their growth rate (follows from Eq. 12):

$$
\begin{aligned}
& \dot{X}^{1}(t)=\dot{X}^{1}-\dot{X}^{0}=\frac{1}{c_{2}^{2}-c_{2}^{1}}\left({ }^{d} J_{2}^{2}-\frac{N_{2}^{2}}{N_{2}^{1}} J_{2}^{1}\right), \\
& \dot{X}^{2}(t)=\dot{X}^{2}-\dot{X}^{1}=\frac{1}{c_{2}^{2}-c_{2}^{1}}\left({ }^{d} J_{2}^{1}-{ }^{d} J_{2}^{2} \frac{\left(1-N_{2}^{1}\right)}{\left(1-N_{2}^{2}\right)}\right) .
\end{aligned}
$$

Unknowns in Eq. (D15) are 4 deviations of compositions at interfaces.

${ }^{1}$ C. Wagner, in Atom Movements (ASM, Cleveland, 1951).

${ }^{2}$ A. M. Gusak and Y. A. Lyashenko, Phys. Metals Metall. 68, 60 (1989).

${ }^{3}$ K. P. Gurov, A. M. Gusak, V. V. Kondrat'yev, and M. V. Yarmolenko, Phys. Metals Metall. 66, 29 (1990).

${ }^{4}$ A. M. Gusak and M. V. Yarmolenko, J. Appl. Phys. 73, 4881 (1993).

${ }^{5}$ M. Hillert and J. Agren, Scr. Mat. 50, 697 (2004).

${ }^{6}$ L. S. Darken, Trans. A.I.M.E. 174, 184 (1948).

${ }^{7}$ U. Gösele and K. N. Tu, J. Appl. Phys. 53, 3252 (1982).

${ }^{8}$ A. M. Gusak and K. P. Gurow, Фииедение (АН СССР) 53, 842 (1982).

${ }^{9}$ A. M. Gusak, F. Hodaj, and A. O. Bogatyrev, J. Phys.: Cond. Matter. 13, 2767 (2001).

${ }^{10}$ A. M. Gusak and G. V. Lucenko, Acta Mater. 46, 3343 (1998).

${ }^{11}$ C. Matano, Jpn. Phys. 8, 109 (1933).

${ }^{12}$ M. Danielewski and B. Wierzba, Phil. Mag. 89, 331 (2009).

${ }^{13}$ M. Danielewski and B. Wierzba, Acta. Mater. 58, 6717 (2010).

${ }^{14}$ The CADiff software web page: http://rcadiff.diffusion.pl.

${ }^{15}$ W. H. Press, B. P. Flannery, S. A. Teukolsky, and W. T. Vetterling, Numerical Recipes in C: The Art of Scientific Computing (Cambridge University Press, 1992).

${ }^{16}$ J. C. Zhao, M. R. Jackson, and L. A. Peluso, Acta Mater. 51, 6395 (2003).

${ }^{17}$ S. Kobayashi and S. Zaefferer, J. Alloys Compounds 452, 67 (2008).

${ }^{18}$ M. Pawelkiewicz, Ph.D. thesis, AGH-University of Science and Technology, Krakow 2011. 
${ }^{19}$ Z. Mei, A. J. Sunwoo, and J. W. Morris, J. Metallurgical Trans. 23A, 857 (1992).

${ }^{20}$ M. Onishi and H. Fujibuchi, Trans. JIM 16, 541 (1975).

${ }^{21}$ Z. Lubyova, P. Fellner, and K. Matiasovsky, Z. Metallkde. 66, 179 (1975).

${ }^{22}$ M. Hillert, Phase Equilibria, Phase Diagrams and Phase Transformations: Their Thermodynamic Basis (Cambridge Univ. Press, 1998).

${ }^{23}$ K. Holly and M. Danielewski, Phys. Rev. B 50, 13336 (1994).
${ }^{24}$ J. H. Shim, C. S. Oh, B.-J. Lee, and D. N. Lee, Z. Metallkd. 87, 205 (1996).

${ }^{25}$ O. Knacke, O. Kubashewski, and K. Hesselmann, Thermochemical Properties of Inorganic Substances, 2nd ed. (Springer, Berlin, 1991).

${ }^{26} \mathrm{M}$. Oh, Ph.D. thesis, Lehigh University, 1994.

${ }^{27}$ K. F. Dreyer, W. K. Neils, R. R. Chromik, D. Grosman, and E. J. Cotts, App. Phys. Lett. 67, 2795 (1995). 